\title{
Beiträge zur Kenntnis der Glyceride der Fette und öle.
}

\section{Weitere Anwendungen der Schmelzpunkts-Differenz in der Fettanalyse.}

Von

\author{
A. Bömer in Münster i. W.
}

Nach in Gemeinschaft mit R. Krönig, R. Ley and H. Merten ausgeführten Untersuchungen.

Das vor kurzem ${ }^{3}$ ) beschriebene neue Verfahren zum Nachweise von Talg in Schweinefett, das auf den Unterschieden in den Schmelzpunkts-Differenzen zwischen den schwerlöslichen Glyceriden und den aus diesen gewonnenen Fettsäuren beim Schweinefett einerseits und bei den Talgen andererseits beruht, haben wir im Anschlusse an die bereits veröffentlichten Untersuchungen noch bei einer Reihe weiterer Fette und Fettmischungen, sowie bei gehärteten Ölen und bei Gemischen von Sehweinefett mit diesen angewendet; ferner wurde das Verfahren auch bei sog. anormalen Schweinefetten geprüft.

Im Anschlusse an den Bericht über das neue Verfahren auf der Hauptversammlung des Vereins Deutscher Nahrungsmittelchemiker in Breslau wurde ferner bereits kurz bemerkt, daß das Prinzip dieses Verfahrens zum Nachweise von Schweinefett in Butter und Gänsefett verwendbar zu sein acheine; auch in dieser Richtung haben wir inzwischen einige Versuche ausgeführt.

Über die Ergebnisse aller dieser Untersuchungen soll im nachfolgenden berichtet werden. Sämtliche Schmelzpunkte sind korrigiert.

\section{Weitere Untersuchungen iiber den Nachweis von Talgen in Schweinefetten.}

(Nach in Gemeinschaft mit R. Krönig ausgeführten Untersuchungen.)

1. Nachweis von Talg in Gemischen von Schweinefett mit Pflanzenfetten und -ölen.

Nachdem wir durch die Untersuchung von reinen Schweinefetten und Talgen sowie deren Gemischen festgestellt hatten, dab ein Zusatz von Talg zu Schweinefetten durch die Schmelzpunkts-Differenz der schwerlöslichen Glyceride und ihrer Fettsäuren nachgewiesen werden kann, handelte es sich um die weitere Frage, ob dieser Nachweis auch möglich ist, wenn neben dem Talg noch Pflanzenfette und -öle in den Schweinefetten vorhanden sind. Unsere Kenntnisse über die schwerlöslichsten Glyceride der Pflanzenfette und -öle und deren Eigenschaften sind noch sehr lückenhaft, und da es nicht möglich war, ohne langwierige Untersuchungen die Natur der schwerlöslichsten Glyceride der gebräuchlichsten Pflanzenfette und -öle festzustellen, begnügten wir uns einstweilen mit einem praktischen Versuch an selbsthergestellten Gemischen von gleichen Teilen Schweinefett und den betreffenden Pflanzenfetten und ölen.

$\mathrm{Zu}$ diesen Versuchen diente das bereits mehrfach verwendete Schweine-Flomenfett $\mathrm{XV}^{2}$ ), aus dem Gemische mit je 50\% Cocosfett, Erdnußöl, Sesamöl und Baumwollsamenöl hergestellt und untersucht wurden.

1) Diese Zeitsehrift 1913, 26, 559-618.

2) Diese Zeitschrift 191:3, 266, 599 and 601 . 
Je $100 \mathrm{~g}$ dieser Fett-Gemische wurden zunächst aus $100 \mathrm{ccm}$ einer Mischung von 4 Teilen Äther und 1 Teil 95 vol.- $\%$-igem Alkohol und dann zweimal aus je $50 \mathrm{ccm}$ Äther krystallisiert. Von den aus den beiden letzten Krystallisationen gewonnenen Glyceriden und ihren Fettcäuren wurden die Schmelzpunkte bestimmt. Ferner stellten wir ein Gemisch aus 2 Teilen des Flomenfettes XV, 2 Teilen des obigen Cocosfettes und je 1 Teile des obigen ErdnuBöles und Baumwollsamenöles her und krystallisierten $50 \mathrm{~g}$ davon zunächst zweimal aus je $50 \mathrm{ccm}$ Aceton und darauf zweimal aus je 50 ccm Äther. Die gewonnenen Ergebnisse, denen auch die bei dem reinen Flomenfette $X V$ früher erhaltenen nochmals beigefügt sind, waren folgende:

\begin{tabular}{|c|c|c|c|c|c|c|c|}
\hline $\begin{array}{l}\text { Glycerid- } \\
\text { Schmelzp. } \\
\quad(\mathrm{Sg})\end{array}$ & $\begin{array}{l}\text { Fett- } \\
\text { säuren- } \\
\text { Schmelzp. } \\
\text { (Sf) }\end{array}$ & $\begin{array}{l}\text { Schmelzp.* } \\
\text { Differenz } \\
\text { (d) }\end{array}$ & $S g+2 d$ & $\begin{array}{l}\text { Glycerid- } \\
\text { Schmelzp. } \\
\quad(\mathrm{Sg})\end{array}$ & $\begin{array}{l}\text { Hett- } \\
\text { säuren- } \\
\text { Schmelzp. } \\
\text { (Sf) }\end{array}$ & $\begin{array}{l}\text { Sebmelzp.- } \\
\text { Differenz } \\
\text { (d) }\end{array}$ & $S g+2 d$ \\
\hline \multicolumn{4}{|c|}{ Flomenfett $X V$. } & \multicolumn{4}{|c|}{ Desgl. mit $50 \%$ Sesamol. } \\
\hline $61,7^{0}$ & 54,00 & 7,70 & 77,1 & 64,30 & 59,30 & 5,00 & 74,3 \\
\hline 63,90 & $58,2^{0}$ & 5,70 & 75,3 & 65,60 & $60,6^{0}$ & $5,0^{0}$ & 一 \\
\hline 65,20 & 60,60 & $4,6^{0}$ & - & \multirow{2}{*}{\multicolumn{4}{|c|}{ Desgl. mit $50 \%$ Baumwolls amenöl. }} \\
\hline $65,4^{9}$ & 61,10 & $4,5^{0}$ & - & & & & \\
\hline \multicolumn{4}{|c|}{ Flomenfett XV mit $50 \%$ Cocosfett. } & $64,6^{0}$ & $59,6^{\circ}$ & $5,0^{0}$ & 74,6 \\
\hline $64,3^{0}$ & 59,10 & $5,2^{0}$ & 74,7 & & & & \\
\hline 65,40 & 60,70 & 4,70 & - & \multicolumn{4}{|c|}{$\begin{array}{l}\text { Gemisch aus Flomenfett XV, Cocosfett, Erd- } \\
\text { nußöl und Baumwollsamenöl. }\end{array}$} \\
\hline \multicolumn{4}{|c|}{ Desgl. mit $50 \% \operatorname{ErdnuBol}$. } & 64,20 & 58,80 & 5,40 & 75,0 \\
\hline $\begin{array}{l}64,3^{0} \\
65,6^{0}\end{array}$ & $\begin{array}{l}59,30 \\
60,80\end{array}$ & $\begin{array}{l}5,0^{0} \\
4,8^{0}\end{array}$ & $\begin{array}{c}74,3 \\
-\end{array}$ & 65,20 & $\begin{array}{l}60,60 \\
61,60\end{array}$ & $4,6^{\circ}$ & - \\
\hline
\end{tabular}

Aus diesen Versuchen geht hervor, daß bei dem starken Zusatze von je $50 \%$ der angewendeten Pflanzenfette und -öle und selbst bei dem zu $2 / 3$ aus Pflanzenfetten und -ölen bestehenden letzten Gemisch die Schmelzpunkts-Differenzen die gleichen waren, wie bei dem zu den Gemischen verwendeten Schweineferte.

Nachdem durch diese Versuche festgestellt war, dab durch die verwendeten Pflanzenfette und -öle die Schmelzpunkts-Differenzen des in den Mischungen vorhandenen Schweinefettes nicht beeinflubt worden waren, prüften wir die weitere Frage, inwieweit bei derartigen Gemischen Talgzusätze noch nachweisbar sind.

Zu diesem Zwecke stellten wir aus dem obigen Gemische von Schweinefett, Cocosfett, Erdnuß- und Baumwollsamenöl (Grundgemisch) 6 weitere Mischungen mit Zusatz von 2,5 und 5\% Rindstalg, Hammeltalg und PreBtalg ${ }^{1}$ ) her. 30 bezw. $50 \mathrm{~g}$ der Gemische wurden mehrmals aus 50 bezw. $100 \mathrm{ccm}$ Aceton und zuletzt aus 25 bezw. $50 \mathrm{ccm}$ Ather krystallisiert; von der zweiten Krystallisation an wurden die Schmelzpunkte bestimmt. Die bei diesen Gemischen erhaltenen Ergebnisse waren folgende:

1) Es waren dies Rindstalg I, Hammeltalg III und Preftalg IV, die bereits zu den früheren Versuchen (Diese Zeitschrift 1913, 26, 601) gedient hatten. 
Grundgemisch mit Zusatz von

\begin{tabular}{|c|c|c|c|c|c|c|c|}
\hline \multicolumn{4}{|c|}{$2,5 \%$ Talg } & \multicolumn{4}{|c|}{$5 \%$ Talg } \\
\hline $\begin{array}{l}\text { Glycerid- } \\
\text { Schmelzp. } \\
\text { (Sg) }\end{array}$ & $\begin{array}{c}\text { Fett- } \\
\text { säuren- } \\
\text { Schmelzp. } \\
(\mathrm{Sf})\end{array}$ & $\begin{array}{l}\text { Schmelzp.- } \\
\text { Differenz } \\
\text { (d) }\end{array}$ & $\mathrm{Sg}+2 \mathrm{~d}$ & $\begin{array}{l}\text { Glycerid- } \\
\text { Schmelzp. } \\
\text { (Sg) }\end{array}$ & $\begin{array}{l}\text { Fett- } \\
\text { säuren. } \\
\text { Schmelzp. } \\
\text { (Sf) }\end{array}$ & $\begin{array}{l}\text { Schmelzp.- } \\
\text { Differenz } \\
\text { (d) }\end{array}$ & $\mathrm{Sg}+2 \mathrm{~d}$ \\
\hline \multicolumn{8}{|c|}{ Rindstalg } \\
\hline 62,30 & $57,5^{0}$ & 4,80 & 71,9 & $61,3^{17}$ & 57,00 & 4,30 & 69,9 \\
\hline 63,30 & $58,5^{0}$ & 4,80 & 72,9 & 62,60 & 58,70 & 3,90 & 70,4 \\
\hline $6 \frac{4}{4}, 2^{\circ}$ & 60,60 & $3,6^{0}$ & 71,4 & $63,6^{0}$ & 60,20 & 3,40 & 70,4 \\
\hline 64,90 & 61,70 & 3,20 & 71,3 & $64,3^{0}$ & 61,30 & 3,00 & 70,3 \\
\hline & & & & $65,0^{0}$ & $62,3^{\circ}$ & 2,70 & 70,4 \\
\hline \multicolumn{8}{|c|}{ Hammeltalg } \\
\hline 60,00 & 58,10 & 1,90 & - & 59,90 & 58,40 & $1,5^{0}$ & - \\
\hline 60,80 & $60,0^{\circ}$ & 0,80 & - & 61,30 & 61,30 & 00 & 61,3 \\
\hline & & & & $62,4^{0}$ & 62,30 & 0,10 & 62,6 \\
\hline \multicolumn{8}{|c|}{ Prebtalg } \\
\hline 59,70 & 57,50 & 2,20 & - & $59,7^{\circ}$ & 58,10 & $1,6^{0}$ & - \\
\hline 60,20 & 59,50 & 0,70 & - & 60,10 & 59,10 & 1,00 & 一 \\
\hline $62,0^{\circ}$ & 61,70 & 0,30 & 62,6 & 61,90 & $61,8^{\circ}$ & 0,10 & 62,1 \\
\hline
\end{tabular}

Hiemach waren die zugesetzten 2,5\% Rindstalg in dem Grundgemisch aus Schweinefett, Cocosfett, Erdnuß- und Baumwollsamenöl nicht nachweisbar, alle anderen Talgzusätze dagegen kamen in der erniedrigten Schmelzpunkts-Differenz zum Ausdruck.

Die im ersten Augenblicke auffällige Erscheinung, daß in dem obigen Grundgemisch schon $5 \%$ Rindstalg nachweisbar waren, während bei dem in dem Grundgemisch vorhandenen Flomenfette XV nach den früheren Untersuchungen ${ }^{1)}$ selbst $10 \%$ desselben Rindstalges noch nicht nachweisbar waren, erklärt sich offenbar dadurch, daß die Nachweisbarkeit des Talges in diesen Pflanzenfett und -öle enthaltenden Gemischen nicht von dem prozentualen Gehalte an Talg in der Gesamt-Mischung abhängt, sondern lediglich von dem Verhältnisse des Talges zu dem in dem Gemisch vorbandenen Schweinefett, Da dessen Menge in dem Grundgemisch $331 / \mathrm{s} \%$ betrug, so entspricht ein Zusatz von $5 \%$ Talg in 100 Teilen des Grundgemisches einem Zusatz von $15,8 \%$ Talg in dem pflanzenfrei gedachten Schweinefett-Talg-Gemische; ein solcher Talggehalt wäre jedenfalls auch in dem Flomenfette XV selbst nachweisbar gewesen.

Aus den Ergebnissen der vorstehenden Versuche mit Pflanzenfette und -öle enthaltenden Fettgemischen geht hervor, daß die Anwesenheit von Cocosfett, Erdnuß-, Sesam- und Baumwollsamenöl in dem Schweinefette XV den Nachweis von Talg nach dem neuen Verfahren nicht beeinträchtigt hat. Inwieweit diese Beobachtung auch bei anderen Pflanzenfetten und -ölen zutrifft, muR durch weitere Untersuchungen festgestellt werden; wahrscheinlich werden sich die pflanzlichen Öle wohl sämtlich ähnlich verhalten wie die von uns untersuchten Pflanzenöle, dagegen erscheint es sehr fraglich, ob auch die natürlichen festen Pflanzenfette, wie z. B. die Mowrah- und die Sheabutter, ebensowenig den Nachweis von Talg stören wie die genannten Öle. Es 
wurde aber schon in der ersten Mitteilung ${ }^{1}$ ) über das neue Verfahren darauf hingewiesen, daß es leicht mit der Phytosterinacetat-Probe auf Pflanzenfette verbunden werden kann; infolgedessen kann man im gegebenen Falle sich durch die Phytosterinacetat-Probe ein Urteil darüber versehaffen, ob etwaige abweichende Schmelzpunkts-Differenzen auf tierische oder pflanzliche Fette oder Öle zurückzuführen sind; sind pflanzliche Fette oder Öle vorhanden, so bleibt natiurlich noch die Möglichkeit bestehen, daß eine abweichende Schmelzpunkts-Differenz auch durch neben den pflanzlichen Fetten und Ölen vorhandenen Talg oder ein sich diesem ähnlich verhaltendes anderes tierisches Fett verursacht sein kann.

2. Schmelzpunkts-Differenzen von gehärteten Ölen und über deren Nachweis in Schweinefett.

Bei dem großen Interesse, welches die gehärteten Öle nicht nur in technischer sondern auch in analytischer Hinsicht beanspruchen, lag es nahe, sie nach dem neuen Verfahren zu untersuchen und insbesondere auch festzustellen, wie sie sich in Gemischen mit Schweinefett bei dem Nachweise von Talg verhalten. Da bei dem Härtungsprozesse der Öle große Mengen von Glyceriden gesättigter Fettsäuren gebildet werden, so war von vornherein anzunebmen, dab ihre Gegenwart die Nachweisbarkeit von Talg in Schweinefett mehr oder weniger in Frage stellte. Wir haben hierauf schon in der ersten Veröffentlichung ${ }^{1}$ ) über das neue Verfahren hingewiesen.

Zur Untersuchung gelangte je eine Probe von gehärtetem Baumwollsamenöl, Erdnußöl und Sesamö $1^{2}$ ); diese wurden zunächst drei- bis viermal aus Aceton und dann so oft aus Äther umkrystalisiert, als sich noch GlyceridKrystalle aus der Lösung abschieden.

Die Ergebnisse dieser Untersuchungen sind in der nachfolgenden Übersicht zusammengestellt, zu der bemerkt sei, daß die erste Zahlenreihe sich beim Erdnuß- und Sesamöl auf die zweite, beim Baumwollsamenöl dagegen auf die dritte Krystallisation bezieht.

\begin{tabular}{|c|c|c|c|c|c|c|c|c|}
\hline Bar & amwollsam & enöl. & & Erdnußöl. & & & Sesamöl. & \\
\hline $\begin{array}{c}\text { Glycerid. } \\
\text { Schmelzp. } \\
\text { (Sg) }\end{array}$ & $\begin{array}{c}\text { Fett- } \\
\text { säuren- } \\
\text { Schruelzp. } \\
\text { (Sf) }\end{array}$ & $\begin{array}{l}\text { Schmelzp. } \\
\text { Differenz } \\
\text { (d) }\end{array}$ & $\begin{array}{l}\text { Glycerid- } \\
\text { Sehmelzp } \\
\text { (Sg) }\end{array}$ & $\begin{array}{c}\text { Fett- } \\
\text { säuren- } \\
\text { Schmelzp. } \\
\text { (Sf) }\end{array}$ & $\begin{array}{l}\text { Schmelzp. } \\
\text { Differenz } \\
\text { (d) }\end{array}$ & $\begin{array}{l}\text { Glycerid- } \\
\text { Schmelzp } \\
(\mathrm{Sg})\end{array}$ & $\begin{array}{c}\text { Fett- } \\
\text { säuren- } \\
\text { Schmelzp. } \\
\text { (Sf) }\end{array}$ & $\begin{array}{l}\text { Schmelzp.- } \\
\text { Differenz } \\
(d)\end{array}$ \\
\hline 53,10 & 49,50 & 3,60 & 59,70 & 59,30 & 0,40 & 60,70 & 60,30 & 0,40 \\
\hline 54,80 & 51,30 & 8,50 & 60,70 & 60,60 & 0,10 & 62,20 & $61,4^{\circ}$ & 0,80 \\
\hline 55,00 & 51,30 & $3,7^{\circ}$ & $62,5^{0}$ & $62,5^{\circ}$ & 0 & 63,50 & $63,0^{0}$ & 0,50 \\
\hline 57,30 & 54,00 & $3,3^{0}$ & 64,00 & $63,5^{0}$ & 0,50 & 64,70 & 64,10 & $0,6^{0}$ \\
\hline 61,30 & $58,5^{\circ}$ & 2,80 & 65,30 & 64,70 & $0,6^{0}$ & $66,4^{0}$ & 65,30 & 1,10 \\
\hline & & & $66,3^{\circ}$ & 65,60 & 0,70 & 67,80 & $66,0^{\circ}$ & 1,80 \\
\hline & & & 67,20 & 66,40 & 0,80 & 68,90 & $66,6^{\circ}$ & 2,30 \\
\hline & & & 67,80 & 66,60 & 1,20 & 69,60 & 67,40 & 2,80 \\
\hline & & & $68,5^{0}$ & 67,20 & 1,30 & 70,10 & 68,00 & 2,10 \\
\hline & & & 68,80 & $67,5^{0}$ & 1,30 & 70,80 & 68,30 & 2,50 \\
\hline & & & $69,5^{0}$ & $68,2^{\circ}$ & 1,30 & $71,5^{\circ}$ & $68,5^{\circ}$ & 3,00 \\
\hline & & & 70,40 & 68,50 & 1,90 & & & \\
\hline & & & 70,60 & $68,6^{\circ}$ & 2,00 & & & \\
\hline
\end{tabular}

1) Diese Zeitschrift $1913,26,607$.

2, Es waren dies die bereits früher (Diese Zeitschreift 1912, 24, 107) untersuchten Proben, und zwar Baumwollsamenöl No. 9, Erdnuß̧öl No. 5 und Sesamöl No. 7. 
Wie aus diesen Zahlen ersichtlich ist, sind die Schmelzpunkte der schwerlöslichsten Glyceride beim gehärteten ErdnuBöl und Sesamöl sebr hoch $\left(70,6^{01}\right)$ und $\left.71,5^{\circ}\right)$; das gleiche ist der Fall bei den Schmelzpunkten der Fettsäuren $\left(68,6^{\circ}\right.$ und $\left.68,5^{\circ}\right)$. Es scheint daher das schwerlöslichste Glycerid dieser beiden gehärteten Öle aus Tristearin zu bestehen.

Das gehärtete Baumwollsaatmehl dagegen zeigt als schwerlöslichstes Glycerid bezw. Glycerid-Gemisch ein solches vom Schmelzpunkte $61,3^{\circ}$, dessen Fettsäuren bei $58,5^{\circ}$ schmelzen. Vermutlich hängen diese niedrigen Schmelzpunkte damit zusammen, daß das verwendete Öl überhaupt nur wenig gehärtet war; sein Schmelzpunkt war $38,5^{0}$.

Die Schmelzpunkts-Differenzen des gehärteten Erdnuß- und Sesamöles sind in den für den Nachweis von Talg in Schweinefett vorwiegend in Betracht kommenden Glycerid-Schmelzpunkts-Intervallen $61-65^{0}$, wie obige Ubersicht zeigt, sehr niedrig $\left(0-0,6^{0}\right.$ bzw. $\left.0,4-0,8^{0}\right)$; sie verhalten sich also ähnlich wie die Schmelz. punkts-Differenzen der Talge. Bei dem Baumwollsamenöl ist die SchmelzpunktsDifferenz des schwerlöslichen Glycerides $\left(61,3^{0}\right)$ zwar etwas größer $\left(2,8^{0}\right)$ als beim Erdnuß- und Sesamöl, immerhin ist sie aber doch noch wesentlich kleiner als bei den Schweinefettglyceriden von gleichem Schmelzpunkt.

Nach diesen Befunden war anzunehmen, daß sich die gehärteten Baumwollsamenöle, Erdnußöle und Sesamöle in Gemischen mit Schweinefett, hinsichtlich des Einflusses auf die Schmelzpunkts-Differenzen ähnlich verhalten wie die Talge. Um diese Frage zu prüfen, wurden Gemische von dem Schweine-Flomenfett XV mit je $50 \%$ der genannten gehärteten Ole und ferner noch zwei solche Gemische mit $50 \%$ gehärtetem Cocosfett nnd Tran hergestellt. Je $50 \mathrm{~g}$ dieser Gemische wurden in der üblichen Weise so oft aus $50 \mathrm{~cm}$ Äther umkrystallisiert, bis sich keine Glyceride mehr ausschieden. Die Ergebnisse dieser Untersuchungen sind in der nachfolgenden Übersicht zusammengestellt.

Gemische ron Schweinefett mit $50 \%$ gehärteten Ölen.

\begin{tabular}{|c|c|c|c|c|c|c|c|}
\hline $\begin{array}{c}\text { Glycerid- } \\
\text { Schmelzp. } \\
\text { (Sg) }\end{array}$ & $\begin{array}{c}\text { Fett- } \\
\text { säuren- } \\
\text { Schmelzp. } \\
\text { (Sf) }\end{array}$ & $\begin{array}{l}\text { Schmelzp.- } \\
\text { Differenz } \\
\text { (d) }\end{array}$ & $\mathrm{Sg}+2 \mathrm{~d}$ & $\begin{array}{l}\text { Glycerid- } \\
\text { Schmelzp. } \\
\text { (Sg) }\end{array}$ & $\begin{array}{c}\text { Fett- } \\
\text { säuren- } \\
\text { Schmelzp. } \\
\text { (Sf) }\end{array}$ & $\begin{array}{l}\text { Schmelzp.- } \\
\text { Differenz } \\
\text { (d) }\end{array}$ & $S g+2 d$ \\
\hline \multicolumn{4}{|c|}{ Flomenfett XV mit gehärtetem Tran. } & \multicolumn{4}{|c|}{ Flomenfett $X V$ mit gehärtetem Exdnuföl. } \\
\hline 58,30 & $56,8^{\circ}$ & 1,50 & -- & 60,00 & 61,60 & $-1,6^{0}$ & - \\
\hline $60,1^{0}$ & 59,40 & 0,70 & - & 61,90 & 62,40 & $-0,50$ & 60,9 \\
\hline 61,30 & 61,70 & $-0,4^{0}$ & 60,5 & $62,6^{0}$ & 63,70 & $-1,10$ & 61,4 \\
\hline 62,90 & 63,40 & $-0,50$ & 61,9 & $63,7^{\circ}$ & 64,90 & $-1,20$ & 61,3 \\
\hline 64,30 & 63,80 & 0,50 & 65,3 & 64,40 & 65,10 & $-0,70$ & 63,0 \\
\hline \multicolumn{4}{|c|}{ Flomenfett XV mit gehärtetem Cocosfett. } & $\begin{array}{l}65,30 \\
66,30\end{array}$ & $65,6^{\circ}$ & $-0,30$ & - \\
\hline 63,20 & $61,8^{\prime \prime}$ & $1,4^{0}$ & 66,0 & $67,4^{0}$ & 66,70 & 0,70 & - \\
\hline $64,2^{0}$ & $63,6^{0}$ & $0,6^{0}$ & 65,4 & $68,5^{0}$ & 67,30 & 1,20 & $\ldots$ \\
\hline $65,5^{0}$ & 64,60 & 0,90 & 65,4 & 69,30 & $67,2^{\circ}$ & 2,10 & - \\
\hline
\end{tabular}

Flomenfett XV mit gehärtetem Baumwoll. samenöl

\begin{tabular}{c|c|c|c|c|c|r|r}
62,60 & 56,50 & 6,10 & 74,8 & 61,30 & 61,40 & $-0,10$ & 61,1 \\
$64,3^{0}$ & 60,40 & 3,90 & 72,1 & 63,60 & 63,30 & 0,30 & 64,2 \\
$65,3^{0}$ & 61,30 & 4,00 & - & 65,10 & 64,50 & 0,60 & -
\end{tabular}

1) Bei diesem Erdnufäl wurde bei der ersten Untersuchung (Diese Zeitschrift 1912, 24, 110) als unlöslichstes Glycerid ein solches vom Schmelzp. $71,0^{\circ}$ (korrig. $71,5^{\circ}$ ) gefunden, dessen Fettsäuren bei $68,6^{\circ}$ (korrig. $69,1^{\circ}$ ) schmolzen. 
Aus diesen Untersuchungsergebnissen ersieht man, daß sich die SchmelzpunktsDifferenzen bei Mischungen von Schweinefett mit gehärtetem Erdnuß- und Sesamöl sowie mit gehärtetem Cocosfett und Tran ähnlich verbalten wie bei Mischungen von Schweinefett mit Talgen, ja die Befunde zeigen sogar, daß diese gehärteten Fette und Öle die Schmelzpunkts-Differenzen anscheinend noch stärker herabdrücken als Zusätze von Talg. Bei dem Gemisch mit 50\% gehärtetem Eranußöl sind sogar sämtliche zu dem Glycerid-Schmelzpunkts-Intervallen $61-65^{\circ}$ gehörigen Schmelzpunkts-Differenzen negativ.

Dagegen ist die Beeinflussung der Schmelzpunkts-Differenzen durch das gehärtete Baumwollsamenöl, wie bei der geringen Härtung des verwendeten Öles von vornherein zu erwarten war, nur gering.

Man wird daher in Zukunft bei Schweinefetten mit erniedrigten SchmelzpunktsDifferenzen damit rechnen müssen, daß diese Abweichungen nicht nur durch Talgzusatz, sondern auch durch den Zusatz von gehärteten pflanzlichen Fetten und Ölen sowie durch gehärteten Tran bedingt sein können, und daher, um ein möglichst zuverlässiges Urteil über das dem Schweinefette zugesetzte Fremdfett zu gewinnen, in der ersten Mutterlauge nach dem Abdestillieren des Lösungsmittels die Prüfung auf Pflanzenfette mittels der Phytosterinacetat-Probe vornehmen.

Wir haben also bei der Prüfung von Schweinefett auf fremde Fette mittels der Schmelzpunkts-Differenz und der Phytosterinacetat-Probe folgende Fälle zu unterscheiden:

I. Die Schmelzpunkts-Differenz ist normal. In diesem Falle liegt entweder ein reines Schweinefett oder ein Gemisch von Schweinefett mit pflanzlichen Fetten oder Ölen vor; hierüber entscheidet die Phytosterinacetat-Probe.

II. Die Schmelzpunkts.Differenz ist erniedrigt (Sg $+2 \mathrm{~d}$ ist kleiner als 71). Dann liegen folgende Möglichkeiten vor:

1. Die Phytosterinacetat-Probe fällt negativ aus: Schweinefett mit Zusatz von Talg, gehärtetem Tran oder von beiden.

2. Die Pbytosterinacetat-Probe fält positi aus. Dann liegen 3 Möglichkeiten vor:
a) Zusatz von gehärteten Pflanzenfetten bezw. -ölen,
b) " "Talg und Pflanzenfetter bezw. -ölen,
c) " " " gehärteten Pflanzenfetten bezw. -ölen.

3. Schmelzpunkts-Differenzen bei sogenannten anormalen Schweinefetten.

$\mathrm{Zu}$ den bisherigen Untersuchungen über den Nachweis von Talg in Schweinefett mittels der Schmelzpunkts-Differenz wurden ausschlieblich Fette von Schweinen verwendet, welche im normalen landwirtschaftlichen Betriebe gewonnen waren.

Da die Zusammensetzung des Schweinefettes aber bekanntlich in mehr oder minder starkem Grade durch das Futter bezw. das Futterfett beeinflußt wird, so war es von besonderem Interesse, auch das Verhalten von solchen Schweinefetten bei dem neuen Verfahren kennen zu lernen, welche unter anormalen, insbesondere einseitigen Fütterungsverbältnissen, gebildet waren. Zu den in dieser Richtung angestellten Versuchen dienten uns die Fette von Schweinen, welche im Jahre 1905/06 in der hiesigen Versuchsstation einseitig gefüttert worden waren; über die Ergebnisse dieser Fütterung haben 
J. König und J. Schluckebier bereits vor längerer Zeit ${ }^{1}$ ) berichtet. Die Fette waren zum Teil bereits vor mehreren Jahren durch Krystallisation aus ÄtherAlkohol von ihren ölsäure- bezw. leinölsäurehaltigen Glyceriden befreit, zum Teil wurden sie aber erst im Laufe des vergangenen Jahres im stark ranzigen Zustande zu der Untersuchung verwendet. Da jedoch, wie wir bereits in der ersten Mitteilung ${ }^{2}$ ) hervorgehoben haben, die Ranzigkeit der Fette von keinem wesentlichen Einflusse auf die Schmelzpunkts-Differenz der Glyceride der gesättigten Fettsäuren zu sein scheint, dürfte die Mitteilung der bei diesen Untersuchungen gewonnenen Ergebnisse doch noch einiges Interesse haben. $\mathrm{Zu}$ den damaligen Fütterungsversuchen dienten je zwei $6-8$ Wochen alte, eben der Muttermilch entwöhnte Ferkel, die abgesehen von etwas Milch, - um die Aufnahme des einseitigen Futters zu erreichen - ausschließlich mit den nachstehend bezeichneten Futtermitteln gefüttert worden waren.

1. Sa ugferkel. Zwei Sangferkel im Gewichte von zusammen $20,06 \mathrm{~kg}$ wurden gleich nach dem Absetzen geschlachtet, um ihr Fett zum Vergleich mit dem durch die weitere Futterung gebildeten Fette zu verwenden.

II. M a is-Fütterung: Die beiden Tiere erhielten - abgesehen von einer Reigabe von etwas Milch an den vier ersten Tagen - 90 Tage lang ausschlieklich Mais schrot, und zwar zusammen in der Zeit vom 28 . Oktober bis 4 . November 1905 täglich $1 \mathrm{~kg}$ und von da an bis zum 22. Januar 1906 täglich 1,5 kg Maisschrot. Ihr Anfangsgewicht betrug 27,1 kg, ihr Endgewicht $39,0 \mathrm{~kg}$.

III. Cocoskuchen-Fütterung: Die beiden Tiere erhielten zunächst sieben Tage lang Kartoffeln und Milch und darauf vom 28. Mai bis 30. Juli 1906 täglich 300 bis $1150 \mathrm{~g}$ Cocoskuchen mit $13 / 4$ bis $1 / 2$ I Milch und vom 31. Juli bis zum 22. Oktober 1906 täglich $1200 \mathrm{~g}$ Cocaskuchen nux mit Wasser angerührt, die dauernd gern aufgenommen wurden. Das Anfangsgewicht beider Schweine zusammen betruy $16,5 \mathrm{~kg}$ und das Endgewicht nach der 144-tägigen Fütterung $64,0 \mathrm{~kg}$.

IV. Banmwollsamenmehl-Fütterung: Da die 6 Wochen alten Tiere das Baumwollsamenmehl selbst beim Anrühren mit Milch nicht aufnehmen wollten, wurde neben dem Baumwollsamenmehl noch Gerstenschrot im Verhältnis 2:1 gegeben und gleichzeitig Baumwollsamenöl in kmulsion hinzugefüttert. Die Menge des Futters betrug am Schlusse des Versuches täglich $600 \mathrm{~g}$ Baumwollsamenmehl $+300 \mathrm{~g}$ Gerstenschrot $+30 \mathrm{~g}$ Baumwollsamenöl. Der Versuch begann am 26. Januar 1906, mußte aber schon bei dem einen Tier am 16. März nnd bei dem anderen 8 Tage später wegen starker Lähnungserscheinungen abgebrochen werden. Das Anfangsgewicht beider Tiere zusammen betrug $20,1 \mathrm{~kg}$ und das Endgewicht nach 50 bezw. 57 Tagen $32,1 \mathrm{~kg}$.

V. Sesammehl-Fütterung: Die beiden Tiere erhielten vom 28. Mai bis 30. Juli 1906 täglich $450-900 \mathrm{~g}$ Sesammehl mit $3 / 4-1 / 2$ l Milch. Da sie bereits nach dieser Fütterung Lähmungserscheinungen zeigten, mußte der Versuch abgeschlossen werden. Das Anfangsgewicht der beiden Tiere zasammen betrug $18,9 \mathrm{~kg}$ and stieg in der 70 -tägigen Periode auf $40,5 \mathrm{~kg}$.

Bei dem Schlachten der Tiere wurden Speck, Kopf-, Schinken-, Bauch- und Flomenfett gesondert und ihre Menge festgestelit.

Die Bestimmung der üblichen analytischen Konstanten für die Fette der verschiedenen Körperteile lieferte folgende Ergebnisse:

1) Diese Zeitschrift 1908, 15, 641. Vergl. auch J. Sehluckebier, Der Einfluf des Futterfettes auf das Körperfett bei Schweinen. - Inaugural-Dissertation. Münster i. W. 1908.

2) Diese Zeitschrift $1913,26,605$. 
A. Bömer, Anwendung der Schmelzpunkts-Differenz. $\left[\begin{array}{l}\text { Zeitsehr. f. Untersuchung } \\ \text { d. Nahr- u, Genumittel. }\end{array}\right.$

\begin{tabular}{|c|c|c|c|c|c|c|}
\hline \multirow[b]{2}{*}{ No. } & \multirow[b]{2}{*}{ Fütterung } & \multirow{2}{*}{$\begin{array}{c}\text { Schmelz- } \\
\text { punkt } \\
\text { o } \mathrm{C}\end{array}$} & \multirow{2}{*}{$\begin{array}{l}\text { Refraktion } \\
\text { des Fettes } \\
\text { bei } 400\end{array}$} & \multicolumn{2}{|c|}{ Jodzahl (pach Wijs) } & \multirow{2}{*}{ Verseifungszahl } \\
\hline & & & & Fett & $\begin{array}{c}\text { Flüssige } \\
\text { Fettsäuren }\end{array}$ & \\
\hline I & Saugferkel & $30,0-31,0$ & $47,6-50,1$ & $60,6-65,9$ & $97,6-101,9$ & $197,2-202,9$ \\
\hline II & Mais & $37,0-47,0$ & $48,4-50,3$ & $59,9-69,7$ & $101,4-104,9$ & $194,9-197,0$ \\
\hline III & $\begin{array}{l}\text { Cocoskuchen } \\
(+ \text { Milch })\end{array}$ & $35,0-38,5$ & $46,5-48,5$ & $37,3-47,5$ & $87,6-89,2$ & $204,6-207,9$ \\
\hline IV & $\begin{array}{l}\text { Baumwollsamen- } \\
\text { mehl ( }+ \text { Gersten- } \\
\text { schrot) }\end{array}$ & $33,0-35,0$ & $58,3-55,9$ & $71,4-79,6$ & $\left.116,4-118,2^{1}\right)$ & $199,0-201,9$ \\
\hline $\mathrm{V}$ & $\begin{array}{l}\text { Sesammehl } \\
(+ \text { Milch })\end{array}$ & $31,5-32,5$ & $53,5-56,0$ & $76,0-78,5$ & $108,5-113,1$ & $195,9-198,6$ \\
\hline
\end{tabular}

Von dem Fette der mit Mais und Cocoskuchen gefütterten Schweine standen noch größere Mengen des Fettes der einzelnen Körperteile, wenngleich im stark ranzigen Zustande, zur Verfügung. Von diesen Fetten wurden daher je $100 \mathrm{~g}$ in der üblichen Weise zunächst dreimal aus $100-200 \mathrm{ccm}$ Aceton und dann so oft aus 50 bis $100 \mathrm{ccm}$ Äther umkrystallisiert, bis die Substanz vollständig verbraucht war bezw. bis sich aus der Lösung keine Glyceride mehr ausschieden. Bei den Mais-Schweinefetten wurden die Schmelzpunkte der Glyceride und ihrer Fettsäuren von der dritten und bei den Cocoskuchen-Schweinefetten von der vierten Krystallisation an bestimmt; die Ergebnisse waren folgende:

\begin{tabular}{|c|c|c|c|c|c|c|c|}
\hline \multicolumn{4}{|c|}{ Mais-Fütterung. } & \multicolumn{4}{|c|}{ Cocoskuchen-Fütterung. } \\
\hline $\begin{array}{c}\text { Glycerid- } \\
\text { Schmelzp. } \\
\text { (Sg) }\end{array}$ & $\begin{array}{l}\text { Fett- } \\
\text { säuren- } \\
\text { Schmolzp. } \\
\text { (Sf) }\end{array}$ & $\begin{array}{l}\text { Schmelzp.- } \\
\text { Differenz } \\
\text { (d) }\end{array}$ & $S g+2 d$ & $\begin{array}{c}\text { Glycerid- } \\
\text { Schmelzp. } \\
\text { (Sg) }\end{array}$ & $\begin{array}{l}\text { Fott- } \\
\text { säuren- } \\
\text { Schmelzp. } \\
\text { (Sf) }\end{array}$ & $\begin{array}{l}\text { Schmelzp. } \\
\text { Differenz } \\
\quad(d)\end{array}$ & $\mathrm{Sg}+2 \mathrm{~d}$ \\
\hline \multicolumn{4}{|c|}{ Flomenfett. } & \multicolumn{4}{|c|}{ Flomenfett. } \\
\hline 62,10 & $55,6^{0}$ & 6,50 & 75,1 & $57,0^{0}$ & 52,00 & $5,0)$. & - \\
\hline $63,5^{0}$ & 57,30 & $6,2^{\circ}$ & 75,9 & $57,4^{0}$ & $52,4^{0}$ & 5,00 & - \\
\hline 64,40 & 58,70 & 5,70 & 75,8 & $58,5^{\circ}$ & 53,50 & 5,00 & - \\
\hline 64,80 & 59,60 & 5,20 & 76,2 & 58,90 & $54,0^{\circ}$ & 4,90 & - \\
\hline 66,20 & 61,30 & 4,90 & - & 59,10 & 54,60 & 4,50 & - \\
\hline $66,4^{0}$ & 61,80 & 4,60 & - & & & & \\
\hline $66,4^{0}$ & $62,2^{0}$ & 4,20 & - & & & & \\
\hline 66,60 & $62,3^{0}$ & 4,30 & - & & & & \\
\hline \multicolumn{4}{|c|}{ Banchett. } & \multicolumn{4}{|c|}{ Bauchfett. } \\
\hline $61,6^{\circ}$ & 55,30 & 6,30 & 74,2 & $57,2^{\circ}$ & $52,2^{\circ}$ & 5,00 & - \\
\hline 64,00 & 58,10 & 5,90 & 75,8 & 58,10 & 52,80 & 5,30 & - \\
\hline $64,5^{0}$ & 59,20 & $5,3^{0}$ & 75,1 & 59,00 & $54,0^{0}$ & $5,0^{\prime}$ & - \\
\hline $65,2^{0}$ & 60,50 & 4,70 & - & $59,3^{\prime}$ & 54,20 & 5,10 & - \\
\hline 65,80 & $61,3^{6}$ & 4,50 & - & 59,50 & 54,60 & 4,90 & - \\
\hline 66,30 & 61,70 & $4,6^{\circ}$ & - & & & & \\
\hline 66,40 & $62,2^{0}$ & $4,2^{\circ}$ & - & & & & \\
\hline
\end{tabular}

1) Nar beim Kopf- und Schinkenfett bestimmt. 


\begin{tabular}{|c|c|c|c|c|c|c|c|}
\hline $\begin{array}{l}\text { Glycerid- } \\
\text { Schmelzp. } \\
\text { (Sg) }\end{array}$ & $\begin{array}{l}\text { Fett- } \\
\text { säuren- } \\
\text { Schmelzp. } \\
\text { (Sf) }\end{array}$ & $\begin{array}{l}\text { Schmelzp.- } \\
\text { Differenz } \\
\text { (d) }\end{array}$ & $\mathrm{Sg}+2 \mathrm{~d}$ & $\begin{array}{l}\text { Gly cerid- } \\
\text { Schmelzp. } \\
\text { (Sg) }\end{array}$ & $\begin{array}{l}\text { Fett- } \\
\text { süuren- } \\
\text { Schmelzp. } \\
\text { (Sf) }\end{array}$ & $\begin{array}{l}\text { Schmelzp.- } \\
\text { Differenz } \\
\text { (d) }\end{array}$ & $S g+2 d$ \\
\hline \multicolumn{4}{|c|}{ Speckfett. } & \multicolumn{4}{|c|}{ Speckfett. } \\
\hline $61,5^{\circ}$ & 55,30 & $6,2^{0}$ & 73,9 & $57,2^{0}$ & 52,20 & 5,00 & - \\
\hline $64,4^{0}$ & 58,80 & $5,6^{0}$ & 75,6 & 57,90 & 53,00 & $4,9^{0}$ & - \\
\hline $65,2^{0}$ & $60,3^{0}$ & 4,90 & - & 59,10 & $53,6^{0}$ & 5,50 & - \\
\hline 66,00 & $61,3^{0}$ & $4,7^{\circ}$ & - & 59,10 & 54,00 & 5,10 & $\longrightarrow$ \\
\hline \multicolumn{4}{|c|}{ Schinkenfett. } & \multicolumn{4}{|c|}{ Schinkenfett. } \\
\hline 62,40 & 55,70 & $6,6^{0}$ & 75,6 & $58,1^{\circ}$ & 53,00 & 5,10 & - \\
\hline $64,4^{0}$ & 58,80 & $5,6^{\circ}$ & 75,6 & 58,30 & 53,40 & $4,9^{\circ}$ & - \\
\hline 64,90 & $59,8^{0}$ & $5,1^{0}$ & 75,1 & 59,10 & 53,90 & 5,20 & $\cdots$ \\
\hline $66,0^{0}$ & $61,4^{0}$ & 4,60 & - & $59,5^{0}$ & 54,20 & 5,30 & $一$ \\
\hline \multicolumn{4}{|c|}{ Kopffett. } & \multicolumn{4}{|c|}{ Kopffett. } \\
\hline $61,2^{0}$ & 55,30 & $5,9^{0}$ & 73,0 & $57,6^{0}$ & $52,4^{\circ}$ & $5,2^{0}$ & - \\
\hline $64,3^{0}$ & $58,3^{0}$ & 6,00 & 76,3 & $58,0^{0}$ & 58,00 & 5,00 & - \\
\hline 65,10 & 60,30 & 4,80 & - & $59,1^{0}$ & 53,80 & 5,30 & - \\
\hline 65,50 & 60,90 & 4,60 & - & 59,10 & 54,20 & 4,90 & - \\
\hline 65,90 & $61,3^{\circ}$ & 4,60 & - & & & & \\
\hline
\end{tabular}

Hiernach zeigen die Fette der mit Mais gefütterten Schweine normale GlyceridSchmelzpunkte und Sehmelzpunkts-Differenzen. Dagegen wurden bei den Fetten der mit Cocoskuchen gefütterten Schweine keine über $59,5^{0}$ schmelzenden Glyceride erhalten; die Schmelzpunkts-Differenzen schwanken zwischen 4,5 und 5,50. Diese ölsäurearmen (Jodzahl 37,3-47,5), sehr harten Schweinefette zeigen daher sehr abnorme Eigenschaften; $\alpha$-Palmitodistearin scheint darin nur in sehr geringen Mengen vorhanden zu sein. Diese abweichenden Eigenschaften dürften aber für den Nachweis eines Talgzusatzes mittels der Schmelzpunkts-Differenz ohne Einflufo sein. In der Praxis wird man derartige Schweinefette wobl überhaupt kaum antreffen, da die Fütterung der Schweine mit derartig großen Mengen von Cocoskuchen nicht üblich und im allgemeinen auch zu teuer ist. Das Fett der Schweine wird in der Praxis vorwiegend aus Stärke gebildet, die in dem Cocoskuchen überhaupt nicht vorhanden ist.

Von den Fetten der Saugferkel und der mit Baumwollsamenmehl und Sesammehl gefütterten Schweinen standen nur noch geringe Mengen für die Untersuchung zur Verfügung, nämlich :

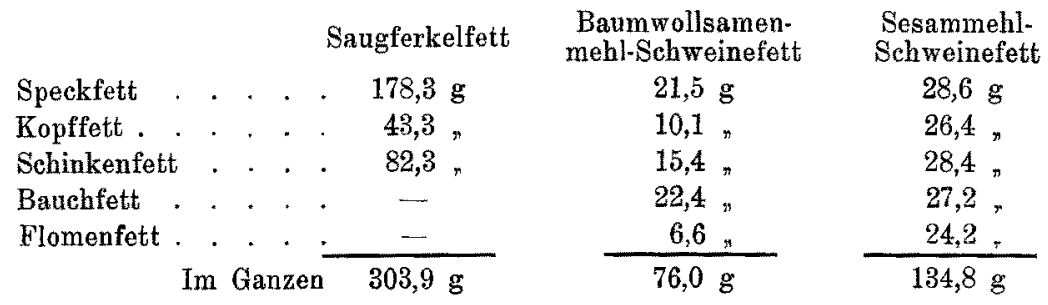

Diese Gesamt-Fette wurden durch mehrmaliges Umkrystallisieren aus ÄtherAlkohol von den ölsäure- und leinölsäurehaltigen, sowie den leichtlöslichen Glyceriden der gesättigten Fettsäuren getrennt, und die auf diese Weise erhaltenen schwerlöslichen 
Glyceride auf ibren Schmelzpunkt und den ihrer Fettsäuren untersucht. Die Ergebnisse dieser Untersuchungen waren folgende:

\begin{tabular}{|c|c|c|c|c|}
\hline & $\begin{array}{l}\text { Glycerid- } \\
\text { Schmelzpunkt } \\
\text { (Sg) }\end{array}$ & $\begin{array}{l}\text { Fettsäuren- } \\
\text { Schmelzpunkt } \\
\text { (Sf) }\end{array}$ & $\begin{array}{l}\text { Sehmelzpunkts- } \\
\text { Differenz } \\
\text { (d) }\end{array}$ & $\mathrm{Sg}+2 \mathrm{~d}$ \\
\hline & $62,2^{0}$ & 56,00 & 6,20 & 76,6 \\
\hline Baumwollsamenmehl-Schweinefett & $63,3^{\circ}$ & 57,10 & 6,20 & 77,7 \\
\hline Sesammehl-Schweinefett & 62,30 & 56,60 & 5,70 & 73,7 \\
\hline
\end{tabular}

Nach vorstehenden Zahlen weichen auch diese Schweinefette in den Schmelzpunkten ihrer schwerlöslichsten Glyceride von normalen Schweinefetten wesentlich ab.

Die erhaltenen Glyceride zeigen Schmelzpunkte, welche wesentlich niedriger sind als der des $\alpha$-Palmitodistearins, des unlöslichsten Glycerides der normalen Schweinefette, mit dem Schmelzpunkt 68,50. Man könnte nun auf den Gedanken kommen, es handele sich bei diesen abnormen Schweinefett-Glyceriden um $\beta$-Palmitodistearin, das ja im reinen Zustande den Sehmelzpunkt $63,3^{\circ}$ hat. Dagegen spricht aber unzweidentig der Schmelzpunkt der Fettsäuren. Dieser liegt beim $\beta$-Palmitodistearin bei $63,2^{0}$, also ungefähr gleich hoch wie der des Glycerides selbst; bei den hier vorliegenden Glyceriden mit den Schmelzpunkten $62,2-63,3^{\circ}$ liegen dagegen die Schmelzpunkte der Fettsäuren wesentlich niedriger, nämlich bei $56,0-57,1^{\circ}$. Das spricht dafür, daß es sich hier um Gemische von Stearodipalmitin mit $\alpha$-Palmitodistearin handelt und daß also auch in den vorliegenden abnormen Schweinefetten $\alpha$-Palmitodistearin, das unlöslichste Glycerid der normalen Schweinefette, vorhanden ist, daß aber seine Menge im Vergleich zum Stearodipalmitin sehr klein ist und daß es daher in den untersuchten Präparaten noch nicht in reiner Form vorliegt.

Für derartige Gemische von Stearodipalmitin und Palmitodistearin sprechen auch die Umwandlungspunkte und die Schmelzpunkte der aus Schmelzfluß erstarrten Glyceride; für diese wurden folgende Werte erhalten:

Glyceride aus

Saugferkelfet

Baumwollsamenmehl-Schweinofett

Sesammehl-Schweinefett .

\section{Sehmolzpunkt der aus Lösung krystallisierten Glycoride} 62,20 63,30 62,30

\section{Umwand- lungspunkt}

49,20

49,70

$49,2^{0}$

Schmelzpunkt
der aus Schmelz-
fluf erstarrten
Glyceride
62,20
63,20
62,20

Die Umwandlungspunkte dieser Glyceride liegen also zwischen denen des Stearodipalmitins $\left(47,1^{\circ}\right)$ und des $\alpha$-Palmitodistearins $\left(51,5^{0}\right)$ der normalen Schweinefette, aber wesentlich niedriger als der des $\beta$-Palmitodistearins $\left(51,6^{\mathbf{0}}\right)$.

Die vorstehenden Untersuchungsergebnisse über die unlöslichsten Glyceride der unter anormaler Fütterung entstandenen Schweinefette sind dahin zusammenzufassen, daß das Saugferkelfett und die durch einseitige Fütterung mit Cocoskuchen, Baumwollsamenmehl und Sesammehl gebildeten Schweinefette in dem Mengenverhältnis und infolgedessen auch in den Schmelzpunkten ihrer unlöslichsten Glyceride bezw. GlyceridGemische von den normalen, vorwiegend aus Stärke gebildeten Schweinefetten mehr oder weniger insofern abweichen, als in jenen Fetten das $\alpha$-Palmitodistearin gegenüber dem Stearodipalmitin zurücktritt, daß aber dem normalen Schweinefette fremde schwerlösliche Glyceride der gesättigten Fettsäuren durch die abnorme Fütterung anscheinend nicht gebildet werden.

Es wird also selbst durch die einseitigste Fütterung von 
Schweinen mit Mais, Cocoskuchen, Baumwollsamenmehl und Sesammehl das Schweinefett nicht derartig verändert, dab dadurch nach dem neuen auf der Schmelzpunkts-Differenz der schwerlöslichsten Glyceride und ihrer Fettsäuren beruhenden Verfahren ein Talggehalt in einem Schweinefette vorgetäuscht werden könnte.

\section{Nachweis von Schweinefett und Talg in Cocosfett.}

Nach in Gemeinschaft mit R. Krönig ausgeführten Untersuchungen.

Aus Anlaß eines besonderen Falles aus der Praxis, in welchem Auskunft darüber gewünscht wurde, ob es möglich sei, nachzuweisen, daß eine angeblich aus Cocosfett bestehende pflanzliche ("koschere") Margarine frei von Schweinefett sei, untersuchten wir zunächst je eine Probe von raffiniertem Cocosfett und Palmkernfett des Handels auf die Schmelzpunkts-Differenzen ihrer unlöslichsten Glyceride; die hierbei erhaltenen Ergebnisse waren folgende:

\section{Cocosfett.}

$50 \mathrm{~g}$ Fett wurden dreimal bei etwa $10^{\circ}$ aus $50 \mathrm{ccm}$ Aceton krystallisiert. Bei der dritten Krystallisation fielen nur noch sehr geringe Glyceridmengen aus, die ein weiteres Umkrystallisieren nicht mehr gestatteten und folgende Werte ergaben:

$\begin{array}{ccc}\text { Glycerid- } & \text { Fettsäuren- } & \text { Schmelzpunkts- } \\ \text { Schmelzpunkt (Sg) } & \text { Schmelzpunkt (Sf) } & \text { Differenz (d) } \\ 47,30 & 44,3^{0} & 3,0^{\circ}\end{array}$

Palmkernfett.

$100 \mathrm{~g}$ Fett wurden zunächst dreimal aus je $100 \mathrm{ccm}$ Aceton krystallisiert; darauf wurden die Schmelzpunkte der ausgeschiedenen Glyceride (I) bestimmt und diese dann noch zweimal aus je $50 \mathrm{ccm}$ Aceton krystallisiert (II und III); bei der letzten Krystallisation wurden nur noch sehr geringe Glyceridmengen gewonnen. Die erhaltenen Ergebnisse waren folgende:

$\begin{array}{cccc} & \begin{array}{c}\text { Glycerid- } \\ \text { Schmelzpunkt (Sg) }\end{array} & \begin{array}{c}\text { Fettsäuren- } \\ \text { Schmelzpunkt (Sf) }\end{array} & \begin{array}{c}\text { Schmelzpunkts- } \\ \text { Differenz }(d)\end{array} \\ \text { I. } & 39,90 & 34,10 & 5,80 \\ \text { II. } & 45,20 & 41,10 & 4,10 \\ \text { III. } & 46,6^{0} & 45,60 & 1,00\end{array}$

Darauf wurden Gemische des Cocosfettes mit 5 und $10 \%$ des SchweineFlomenfettes XV und mit 10\% des Rindstalges $I$ hergestellt und je $50 \mathrm{~g}$ in der gleichen Weise wie das reine Cocosfett dreimal aus je 50 ccm Aceton und alsdann noch ein viertes Mal in derselben Weise aus Aceton bezw. bei der talghaltigen Mischung aus Äther krystallisiert, wobei nur noch geringe Mengen von Glyceriden ausfielen. Der Versuch mit der 10\% Schweinefett enthaltenden Mischung wurde noch ein zweites Mal ausgeführt.

Die Ergebnisse der Schmelzpunktsbestimmungen nach der 3. (T) und 4. Krystallisation (II) waren folgende: 
Cocosfett mit Zasatz von

\begin{tabular}{|c|c|c|c|c|c|c|c|c|c|c|c|}
\hline \multicolumn{4}{|c|}{$5 \%$ Schweinefett. } & \multicolumn{4}{|c|}{$10 \% \mathrm{sch} w e$ inefett. } & \multicolumn{4}{|c|}{$10 \%$ Rindstalg. } \\
\hline $\begin{array}{c}\text { Glycerid-- } \\
\text { Sehmelz- } \\
\text { punkt } \\
\text { (Sg) }\end{array}$ & $\begin{array}{l}\text { Fett- } \\
\text { sãuren- } \\
\text { schmelz- } \\
\text { punkt(Sf) }\end{array}$ & $\begin{array}{l}\text { Schmelz- } \\
\text { punints- } \\
\text { Differenz } \\
\text { (d) }\end{array}$ & $s g+2 d$ & $\begin{array}{l}\text { Glycerid- } \\
\text { Sehmmelz- } \\
\text { punkt } \\
\text { (Sg) }\end{array}$ & $\left|\begin{array}{c}\text { Fett- } \\
\text { säuren- } \\
\text { Schmelz- } \\
\text { punkt (Sf) }\end{array}\right|$ & \begin{tabular}{|} 
Sehmelz- \\
punkts- \\
Differenz \\
(d)
\end{tabular} & $s g+2 d$ & $\begin{array}{l}\text { Glyeerid- } \\
\text { sehmelz- } \\
\text { punkt } \\
\text { (Sg) }\end{array}$ & $\begin{array}{c}\text { Fett- } \\
\text { suluren- } \\
\text { Schmelz- } \\
\text { penkt(Sf) }\end{array}$ & $\begin{array}{l}\text { Sehmelz- } \\
\text { punlkts- } \\
\text { Differenz } \\
\text { (d) }\end{array}$ & $+2 d$ \\
\hline I. $61,3^{\circ}$ & 53,80 & 7,50 & 76,3 & 62,30 & 54,00 & 8,30 & 78,9 & 60,30 & $60,2^{0}$ & 0,10 & - \\
\hline II. 63,30 & 56,30 & 7,00 & 77,3 & 63,50 & $57,4^{0}$ & 6,10 & 75,7 & $64,0^{0}$ & $63,0^{0}$ & 1,00 & 66,0 \\
\hline & & & & Wiede & rbol & 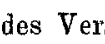 & suches & & & & \\
\hline & & & & 62,70 & 54,60 & 8,10 & 78,9 & & & & \\
\hline & & & & $64,2^{\circ}$ & $59,0^{\circ}$ & 5,20 & 74,6 & & & & \\
\hline
\end{tabular}

Hiernach hatten bei diesen Gemischen mit Schweinefett und Rindstalg die letzten Krystallisationen weil höhere Glycerid-Schmelzpunkte als die des reinen Cocosfettes; es konnte also durch die Bestimmung der Schmelzpunkte der schwerstlöslichen Glyceride der Schweinefett- und Talgzusatz erkannt oder, richtiger gesagt, nachgerviesen werden, daß das Cocosfett nicht rein war.

Betrachten wir ferner die Schmelzpunkts-Differenzen bei den 3 Gemischen, so sehen wir, daß sie bei den Schweinefett enthaltenden Gemischen viel größer — nämlich 5,2 bis $8,3^{\circ}$ - sind als bei dem rindstalghaltigen Gemisch mit $\alpha=0,1$ bis $1,0^{\circ}$; dementsprechend sind auch die Werte für $\mathrm{Sg}+2 \mathrm{~d}$ bei ersteren wesentlich höher $(74,6-78,9)$ als bei dem letzteren $(66,0)$. Wie man sieht, stimmen diese Werte für $\mathrm{d}$ und $\mathrm{Sg}+2 \mathrm{~d}$ gut überein mit den entsprechenden Werten der Schweinefette und Rindstalge selbst, und es wird daher in gewissen Grenzen möglich sein, nachzuweisen, ob in einer angeblich aus Cocosfett bezw. Palmkernfett hergestellten Margarine Schweinefett, Talge oder Gemische von beiden vorhanden sind, was ja für die Beantwortung der Frage, ob eine Margarine koscher ist, von Bedeutung sein kann. Selbstverständlich kann aber in dieser Hinsicht nur dann eine bestimmte Antwort gegeben werden, wenn die Scbmelzpunkte der schwerstlöslichen Glyceride für Cocosfett bezw. Palnkernfett normal sind, wie es bei einer aus Anlaß des oben erwähnten praktischen Falles untersuchten Probe von Pflanzen-Margarine der Fall war. Die aus dieser Márgarine mach drei- bezw. viermaligem Umkrystallisieren erhaltenen schwerlöslichen Glyceride wiesen folgende Schmelzpunkte auf:

$\begin{array}{cccc}\begin{array}{c}\text { No, der } \\ \text { Krystallisation }\end{array} & \begin{array}{c}\text { Glycerid- } \\ \text { Schmelzpunkt (Sg) }\end{array} & \begin{array}{c}\text { Fettsänren- } \\ \text { Schmelzpunkt (Sf) }\end{array} & \begin{array}{c}\text { Schmelzpunkts- } \\ \text { Differenz (d) }\end{array} \\ 3 & 44,60 & 40,70 & 3,90 \\ 4 & 48,60 & 46,90 & 1,70\end{array}$

Sind die Glycerid-Schmelzpunkte aber wesentlich höher als beim reinen Cocosfett und Palmkernfett, so kann dies, wie wir oben (S. 157) gesehen haben, auch durch einen Gehalt an gehärteten Pflanzenölen bedingt sein.

Durch die vorstehenden Ausführungen über die Untersuchung von Cocosfett und Palmkernfett auf ihre schwerlöslichsten Glyceride soll nur ein weiterer Gesichtspunkt für die Verwendbarkeit unseres neuen Verfahrens zur Beantwortung mancherlei Fragen gegeben werden; selbstverständlich müssen noch weitere Untersuchungen, nicht nur an einer größeren Anzahl von Cocosfett- und Palmkernfettproben angestellt werden, sondern es müssen auch noch die sonstigen festen Pflanzenfette, wie Mowrahfett usw., welche heute bereits zur Herstellung von Margarine Verwendung finden, in dieser Richtung untersucht werden. 
Bei einer Probe Mowrahfett des Handels fanden wir z. B. Glycerid-Schmelzpunkte von $61,3-66,3^{\circ}$ und Schmelzpunkts-Differenzen von $-0,7^{\circ}$ bis $+2,1^{0}$.

\section{Anwendung der Schmelzpunkts-Differenz zum Nachweise von Schweine- fett in Butterfett.}

Vorläufige Mitteilung über in Gemeinschaft mit R. Ley ausgeführte Untersuchungen.

Bereits in den Jahren 1904 und 1905 hatte der eine von uns (A. Bömer) in Gemeinschaft mit A. Schemm und G. Heimsoth Untersuchungen über die festen Glyceride des Butterfettes angestellt und in einer Grasbutter (Reichert-MeiBIsche Zahl 26,7, Polenske'sche Zahl 1,4, Verseifungszahl 224,0) als unlöslichstes Glycerid Tristearin vom Schmelzpunkt $71,7^{\circ}$ gefunden; ferner war durch Untersuchung der letzten Mutterlaugen von der Tristearin-Gewinnung auch das Vorkommen von Stearodipalmitin und Palmitodistearin im Butferfette wahrscheinlich gemacht. Von der Veröffentlicbung dieser Ergebnisse wurde damals abgesehen, weil die beiden letzteren Glyceride noch nicht hinreichend rein waren, und zunächst beim Rinds- und Hammeltalg, wo die Verhältnisse offenbar einfacher lagen, die Methodik der Darstellung reiner Glyceride ausgearbeitet werden mußte.

Im Laufe des vergangenen Jahres hat nun vou C. A m berger seine „Beitrüge zur Kenntnis der Glyceride des Butterfettes"1) veröffentlicht; er gibt darin seine Untersuchungsergebnisse bei 3 Butterfetten bekannt, von denen das eine als unlöslichstes Glycerid Tristearin, die anderen beiden dagegen Palmitodistearin enthielten. In dem einen dieser beiden letzteren Butterfette konnte C. A mberger als weiteres Glycerid Stearodipalmitin nachweisen, während die beiden anderen daraufhin nicht untersucht worden waren.

Die von C. A mberger für das Palmitodistearin und das Stearodipalmitin aus seinen Butterfetten angegebenen Schmelzpunkte stimmen gut überein mit denjenigen, welche wir für die gleichen Glyceride aus Hammeltalg gefunden haben, nämlich:

Glyceride aus

Hammeltalg. Butterfett.

$\begin{array}{cc}\text { Palmitodistearin } \\ \begin{array}{cc}\text { Glycerid- } & \text { Fettsäuren- } \\ 63,30 & \text { Schmelzpunkt }\end{array} \\ 62,9-63,10 & 63,20 \\ & 62,4-63,00\end{array}$

\begin{tabular}{|c|c|}
\hline & \\
\hline $\begin{array}{c}\text { Glycerid- } \\
\text { Schmelzpunkt }\end{array}$ & $\begin{array}{c}\text { Fettsäuren- } \\
\text { Schmelzpunkt }\end{array}$ \\
\hline $57,5^{0}$ & 55,70 \\
\hline
\end{tabular}

Hiernach liegt also in dem Palmitodistearin des Butterfettes ebenso wie in dem des Hammeltalges die $\beta$-Verbindung vor.

Ferner geht aus den Untersuchungen Amberger's hervor, daß die Menge der drei Glyceride Tristearin, Palmitodistearin und Stearodipalmitin im Butterfette gering ist, und aus den Unterechieden in der Konsistenz von Butterfett einerseits und Hammeltalg sowie Rindstalg andererseits kann man ja auch wohl ohne weiteres schließen, daß die Menge der drei Glyceride in den Butterfetten wesentlich geringer ist als in den Talgen.

Bei diesen Überlegungen im Zusammenhalt mit den Ergebnissen der vor kurzem veröffentlichten Untersuchungen über den Nachweis von Talg in Schweinefett mittels der Schmelzpunkts-Differenz lag der Gedanke nahe, das Prinzip des neuen Verfahrens auch für den Nachweis von Schweinefett in Butterfett zu verwenden. Während aber

1) Diese Zeitschrift 1913, 26, 65-85. 
beim Schweinefett durch den Talgzusatz die Schmelzpunkts-Differenz kleiner wird, muß natürlich die Veränderung der Schmelzpunkts-Differenz beim Butterfett durch den Schweinefettzusatz im entgegengesetzten Sinne verlaufen, d. h. die SchmelzpunktsDifferenz muB größer werden.

Daß die geringen Mengen Tristearin, welches wenn auch vielleicht nicht in allen so doch in einigen Butterfetten vorkommt, auf die Schmelzpunkts-Differenz der Glyceride von wesentlichem Einflusse sein werden, ist nicht anzunehmen; auch für den Nachweis von Schweinefett in Butterfett wird vorwiegend der Unterschied in den SchmelzpunktsDifferenzen von $\alpha$ - und $\beta$-Palmitodistearin von Bedeutung sein.

Das $\beta$-Palmitodistearin mit dem Schmelzpunkt $63,3^{0}$ wird trotz der geringen Mengen etwa vorhandenen Tristearins in einigen Butterfetten bei der Krystallisation praktisch als das schwerlöslichste Glycerid des Butterfettes anzusehen sein; man wird daher bei diesem Glyceride mit der geringen Schmelzpunkts-Differenz $(0,1)$ des $\beta$-Palmitodistearins $\mathrm{zu}$ rechnen haben.

Aus Mischungen von Butterfett mit Schweinefett wird man dagegen beim Umkrystallisieren als schwerlöslichste Glyceride solche mit über $63,3^{\circ}$ liegenden Schmelzpunkten erhalten; man wird daher gerade diese schwerlöslichsten Glyceride für den Nachweis ron Schweinefett in Butterfett darstellen und auf ihre SchmelzpunktsDifferenz untersuchen müssen.

Um diese Annahmen auf ihre Richtigkeit zu prüfen, wurden zunächst 4 Proben von Molkereibutter ${ }^{1}$ ) nach dem Krystallisationsverfahren auf ihre SchmelzpunktsDifferenz untersucht und hierbei in folgender Weise verfahren:

$100 \mathrm{~g}$ Butterfett wurden zunächst aus $100 \mathrm{ccm}$, dann dreimal aus $200 \mathrm{~cm}$ Aceton und endlich noch einmal aus $100 \mathrm{ccm}$ und zweimal aus $50 \mathrm{ccm}$ Äther krystallisiert; bei der letzten Krystallisation schieden sich auf diese Weise nur noch sehr geringe Glycerid-Mengen ab, die zu einer weiteren Krystallisation nicht hinreichten.

Die auf diese Weise erhaltenen Ergebnisse sind in der nachfolgenden Übersicht zusammengestellt und in der Fig. 1 (S. 167) graphisch zur Darstellung gebracht.

\begin{tabular}{|c|c|c|c|c|c|c|c|}
\hline $\begin{array}{c}\text { No. der } \\
\text { Krystalli- } \\
\text { sation }\end{array}$ & $\begin{array}{l}\text { Glycerid- } \\
\text { Schmelzp. } \\
\text { (Sg) }\end{array}$ & $\begin{array}{l}\text { Fett- } \\
\text { säuren- } \\
\text { Schmelzp. } \\
\text { (Sf) }\end{array}$ & $\begin{array}{l}\text { Schmelzp.- } \\
\text { Differenz } \\
\text { (d) }\end{array}$ & $\begin{array}{l}\text { No. der } \\
\text { Krystalli- } \\
\text { sation }\end{array}$ & $\begin{array}{c}\text { Glycerid- } \\
\text { Schmelzp. } \\
(\mathrm{Sg})\end{array}$ & $\begin{array}{c}\text { Fett- } \\
\text { säuren- } \\
\text { Schmelzp. } \\
\text { (Sf) }\end{array}$ & $\begin{array}{l}\text { Schmelzp.- } \\
\text { Differenz } \\
\text { (d) }\end{array}$ \\
\hline \multicolumn{4}{|c|}{ Butter I (Molkerei Uentrop). } & \multicolumn{4}{|c|}{ Butter IJI (Molkerei Geseke). } \\
\hline 4 & 56,40 & 58,50 & 2,90 & 4 & 57,20 & 54,30 & 2,90 \\
\hline 5 & 56,70 & 54,30 & 3,30 & 5 & 57,10 & $55,0^{0}$ & 2,10 \\
\hline 6 & 60,40 & $57,3^{0}$ & 3,10 & 6 & 60,30 & 58,40 & 1,90 \\
\hline 7 & 61,50 & 59,80 & 1,70 & 7 & 61,50 & 60,70 & $0,8^{0}$ \\
\hline 8 & 61,70 & 61,90 & $-0,20$ & 8 & 62,30 & $61,5^{0}$ & 0,80 \\
\hline \multicolumn{4}{|c|}{ Butter II (Molkerei Lohne). } & \multicolumn{4}{|c|}{ Butter IV (Molkerei Erndtebrïck). } \\
\hline 4 & $56,4^{0}$ & $53,5^{0}$ & 2,90 & 4 & $56,4^{\circ}$ & 53,40 & 3,00 \\
\hline 5 & 57,00 & 54,20 & 2,80 & 5 & 57,23 & 54,10 & 8,10 \\
\hline 6 & 60,40 & 57,30 & 3,10 & 6 & 60,10 & $57,1^{0}$ & 3,00 \\
\hline 7 & $61,5^{0}$ & 59,70 & 1,80 & 7 & $61,5^{0}$ & 59,70 & 1,80 \\
\hline 8 & 62,40 & $62,1^{0}$ & $0,3^{0}$ & 8 & 62,40 & 61,90 & 0,50 \\
\hline
\end{tabular}

1) Die Butterproben wurden anfangs September 1913 gelegentlich einer vom MeiereiVerbande für Westfalen und Lippe veranstalteten Butterprüfung entoommen. 


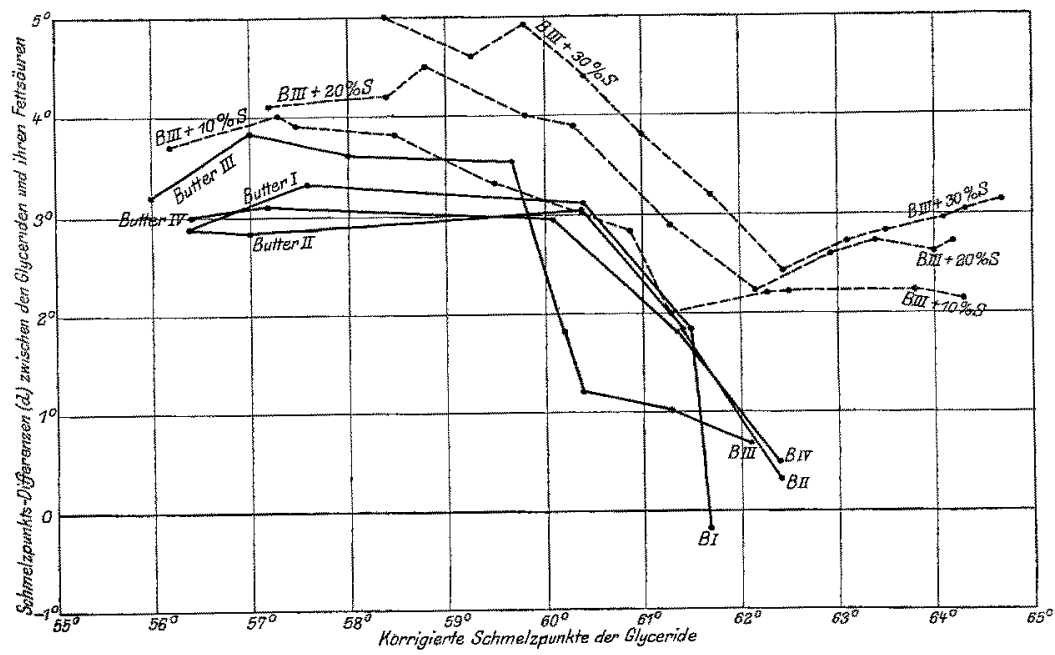

Fig. 1.

Beziehungen $z$ wischen Glycerid-Schmelzpunkten und Schmelzpunkts-Differenzen bei den reinen Butterfetten I-IV und Gemischen des Butterfettes III mit 10, 20 and $30 \%$ Schweinefett.

Aus diesen Untersuchungsergebnissen geht zunächst hervor, daß bei keinem der reinen Butterfette der Glycerid-Schmelzpunkt über $62,4^{0}$ gestiegen ist.

Ferner ersieht man, daß die Schmelzpunkts-Differenzen bei den letzten Krystallisationen sehr gering bezw. sogar negativ werden; dieses rührt daher, daß es sich bei diesen letzten Krystallisationen des Butterfettes um Gemische von Stearodipalmitin und $\beta$-Palmitodistearin (bezw. auch etwas Tristearin) handelt, von denen wir nach den Versuchen mit Gemisehen der reinen Glyceride ${ }^{1}$ ) wissen, daß sie in dem Glycerid-Schmelzpunkts-Intervalle $61-65^{0}$ niedrige Schmelzpunkts-Differenzen aufweisen.

Da somit Aussicht vorhanden war, durch die Veränderung der SchmelzpunktsDifferenzen Schweinefett in Butterfett nachweisen zu können, untersuchten wir eine Reibe selbsthergestellter Mischungen der Butterfette I-IV mit verschiedenen Mengen von Schweinefetten ${ }^{2}$ ).

Erste Versuchsreihe: Je $100 \mathrm{~g}$ der Gemische der Butterfette I und III mit 10 und $20 \%$ des Schweine-Flomenfettes XV wurden zunächst 3-5-mal aus $100 \mathrm{cem}$ Aceton, sodann noch mehrmals aus 50-100 ccm Äther krystallisiert, bis aus der Lösung nur noch geringe Ausscheidungen erfolgten.

Von der 3. Krystallisation an wurden die Sehmelzpunkte der Glyceride und der ans ihnen in der früher beschriebenen Weise dargestellten Fettsäuren bestimmt.

Hierbei wurden folgende Ergebnisse erhalten:

1) Diese Zeitschrift 1913, 28, 589.

2) Die Versuche wurden mit den Schweinefetten XII, XV und XVI angestellt, welche bereits zu den früheren Versuchen (Diese Zeitschrift 1913, 26, 600-601) gedient hatten. 


\begin{tabular}{|c|c|c|c|c|c|c|c|}
\hline $\begin{array}{l}\text { No. der } \\
\text { Krystalli- } \\
\text { sation }\end{array}$ & $\begin{array}{l}\text { Glycerid- } \\
\text { Schmelzp. } \\
\text { (Sg) }\end{array}$ & $\begin{array}{c}\text { Fett- } \\
\text { säuren- } \\
\text { Schmelzp. } \\
\text { (Sf) }\end{array}$ & $\begin{array}{l}\text { Schmelzp.- } \\
\text { Differenz } \\
\text { (d) }\end{array}$ & $\begin{array}{l}\text { No. der } \\
\text { Krystalli- } \\
\text { sation }\end{array}$ & $\begin{array}{l}\text { Glycerid- } \\
\text { Sehmelzp. } \\
\text { (Sg) }\end{array}$ & $\begin{array}{c}\text { Fett- } \\
\text { säuren- } \\
\text { Schmelzp. } \\
\text { (Sf) }\end{array}$ & $\begin{array}{l}\text { Schmelzp } \\
\text { Differenz } \\
\text { (d) }\end{array}$ \\
\hline \multicolumn{4}{|c|}{ Butter I mit $10 \%$ Flomenfett XV. } & \multicolumn{4}{|c|}{ Butter III mit $10 \%$ Flomenfett XV. } \\
\hline 3 & $58,2^{0}$ & 53,20 & 5,00 & 3 & 58,10 & 58,40 & 4,70 \\
\hline 4 & 58,40 & 54,00 & $3,4^{0}$ & 4 & $58,5^{\circ}$ & 54,30 & $4,2^{\circ}$ \\
\hline 5 & $60,3^{0}$ & 56,20 & $4,1^{0}$ & 5 & 60,10 & $56,5^{0}$ & $3,6^{0}$ \\
\hline 6 & 61 & $59,2^{0}$ & 2 , & 6 & 61,60 & 59,50 & 2,10 \\
\hline 7 & 6 & 60,30 & 2 , & 7 & 63,20 & $60,5^{0}$ & 2,70 \\
\hline 8 & 63,60 & 60,40 & $3,2^{0}$ & 8 & 63,60 & $61,4^{0}$ & 3,20 \\
\hline \multicolumn{4}{|c|}{ Desgl. mit $20 \%$ Flomenfett XV. } & \multicolumn{4}{|c|}{ Desgl mit $20 \%$ Flomenfett XV. } \\
\hline 3 & 59,80 & $54,6^{0}$ & 5,20 & 3 & 59,80 & 55,00 & 4,80 \\
\hline 4 & 60,20 & 55,10 & 5,10 & 4 & $60,2^{0}$ & 55,90 & 4,30 \\
\hline 5 & $61,2^{0}$ & $57,4^{0}$ & 3,80 & 5 & 61,10 & $57,2^{0}$ & 3,90 \\
\hline 6 & 62,30 & 59,20 & 3,10 & 6 & 62,50 & 59,50 & 3,00 \\
\hline 7 & 63,30 & 59,90 & 3,40 & 7 & 63,60 & $60,6^{0}$ & 3,00 \\
\hline 8 & 63,90 & $61,1^{0}$ & 2,80 & 8 & $64,4^{0}$ & $61,4^{0}$ & 3,00 \\
\hline 9 & $64,6^{0}$ & 61,20 & 3,40 & 9 & 64,90 & 62,10 & 2,80 \\
\hline
\end{tabular}

Zweite Versuchsreihe. In ähnlicher Weise wie in der ersten Versuchsreihe wurden auch Versuche mit Gemischen der Butterfette II und IV mit 10 und $20 \%$ Flomenfett XII angestellt, jedoch wurden je $100 \mathrm{~g}$ der Fettgemische nur einmal aus $100 \mathrm{ccm}$ Aceton und dann wieder so oft aus 50-100 ccm Äther krystallisiert, bis die Glyceridmenge so gering war, dak sie für ein Umkrystallisieren bezw. für eine nochmalige Verseifung nicht mehr hinreichte.

Die Ergebnisse dieser Versuchsreihe waren folgende:

\begin{tabular}{|c|c|c|c|c|c|c|c|}
\hline $\begin{array}{c}\text { No. der } \\
\text { Krystalli- } \\
\text { sation }\end{array}$ & $\begin{array}{l}\text { Glycerid- } \\
\text { Schmelzp. } \\
\text { (Sg) }\end{array}$ & $\begin{array}{l}\text { Fett- } \\
\text { säuren- } \\
\text { Schmelzp. } \\
\text { (Sf) }\end{array}$ & $\begin{array}{l}\text { Schmelzp.- } \\
\text { Differenz } \\
\text { (d) }\end{array}$ & $\begin{array}{l}\text { No. der } \\
\text { Krystalli- } \\
\text { sation }\end{array}$ & $\begin{array}{c}\text { Glycerid- } \\
\text { Schmelzp. } \\
\text { (Sg) }\end{array}$ & $\begin{array}{c}\text { Fett* } \\
\text { säuren- } \\
\text { Schmelzp. } \\
\text { (Sf) }\end{array}$ & $\begin{array}{l}\text { Schmelzp.- } \\
\text { Differenz } \\
\text { (d) }\end{array}$ \\
\hline \multicolumn{4}{|c|}{ Butter II mit $10 \%$ Flomenfett XII. } & \multicolumn{4}{|c|}{ Batler IV mit $10 \%$ Flomenfett XII. } \\
\hline 2 & 54,20 & 51,20 & 3,00 & 2 & 58,30 & 52,80 & 5,50 \\
\hline 3 & 57,20 & $53,2^{0}$ & 4,00 & 3 & 59,90 & 55,20 & 4,70 \\
\hline 4 & 59,80 & 57,30 & 2,50 & 4 & $61,2^{0}$ & 57,70 & 3,50 \\
\hline 5 & 61,30 & 59,40 & 1,90 & 5 & 62,30 & 59,40 & 2,90 \\
\hline \multirow[t]{2}{*}{$c$} & $62,3^{0}$ & 60,40 & 1,90 & 6 & 62,50 & 60,30 & 2,20 \\
\hline & & & & 7 & 68,40 & $61,2^{0}$ & 2,20 \\
\hline
\end{tabular}

Desgl. mit $20 \%$ Flomenfett XII.

\begin{tabular}{l|l|l|l|}
2 & $56,2^{0}$ & 52,40 & 3,80 \\
3 & $58,9^{0}$ & 55,20 & 3,70 \\
4 & 60,40 & 58,30 & $2,1^{0}$ \\
5 & $62,3^{0}$ & $80,3^{0}$ & 2,00 \\
6 & 63,40 & 61,20 & 2,20 \\
7 & $65,5^{0}$ & 63,40 & $2,1^{0}$
\end{tabular}

Dritte Versuchsreihe: In dieser Versuchsreihe wurden je $100 \mathrm{~g}$ des reinen Butterfettes III sowie von Gemischen dieses Fettes mit 10, 20, $30 \%$ Speckfett XVI zunächst fünfbzw, bei den Gemischen mit 20 und $30 \%$ Speckfett siebenmal aus $100 \mathrm{ccm}$ Aceton und dann 
so oft aus 50-100 ccm Ather umkrystallisiert, bis die Substanz verbraucht war. Die Ergebnisse dieser Versuchsreihe sind in der nachfolgenden Übersicht zusammengestellt und in der Fig. 1 (S. 167) graphisch zur Darstellung gebracht.

\begin{tabular}{|c|c|c|c|c|c|c|c|}
\hline $\begin{array}{l}\text { No. der } \\
\text { Krystalli- } \\
\text { sation }\end{array}$ & $\begin{array}{l}\text { Glycerid. } \\
\text { Schmelzp. } \\
\text { (Sg) }\end{array}$ & $\begin{array}{l}\text { Fett: } \\
\text { säuren- } \\
\text { Schmelzp. } \\
\text { (Sf) }\end{array}$ & $\begin{array}{l}\text { Schmelzp.- } \\
\text { Differenz } \\
\text { (d) }\end{array}$ & $\begin{array}{l}\text { No. der } \\
\text { Krystalli- } \\
\text { sation }\end{array}$ & $\begin{array}{l}\text { Glycerid- } \\
\text { Schmelzp. } \\
\text { (Sg) }\end{array}$ & $\begin{array}{l}\text { Fett- } \\
\text { sẫuren- } \\
\text { Schmelzp. } \\
\text { (Sf) }\end{array}$ & $\begin{array}{l}\text { Schmelzp. } \\
\text { Differenz } \\
\text { (d) }\end{array}$ \\
\hline \multirow{2}{*}{\multicolumn{4}{|c|}{ Butter III. }} & \multicolumn{4}{|c|}{ Desgl. mit $20 \%$ Speckfett XVI. } \\
\hline & & & & 3. & 57,20 & 53,10 & 4,10 \\
\hline 3. & 53,40 & 51,70 & 1,70 & 4. & 58,40 & $54,2^{3}$ & 4,20 \\
\hline 4. & $54,2^{0}$ & 52,20 & 2,00 & 5. & 58,80 & $54,3 v$ & 4,50 \\
\hline 5. & 56,00 & 52,80 & 3,20 & 6. & 59,80 & 55,80 & 4,00 \\
\hline 6. & $57,0^{0}$ & 53,20 & 3,80 & 7. & 60,30 & 56,40 & 5,90 \\
\hline 7. & 58,00 & 54,40 & 3,60 & 8. & $61,3^{0}$ & 58,40 & 2,90 \\
\hline 8. & 59,70 & 56,20 & $3,5^{0}$ & 9. & $62,2^{0}$ & $60,0^{\circ}$ & 2,20 \\
\hline 9. & 60,20 & $58,4^{0}$ & 1,80 & 10. & 62,90 & $60,3^{0}$ & 2,60 \\
\hline 10. & 60,40 & 59,20 & 1,20 & 11. & 63,40 & 60,70 & 2,70 \\
\hline 11. & $61,3^{0}$ & $60,3^{0}$ & 1,00 & 12. & $64,0^{\circ}$ & $61,4^{0}$ & $2,6^{\circ}$ \\
\hline 12. & 62,10 & $61,4^{0}$ & 0,70 & 13. & $64,2^{\circ}$ & $61,5^{0}$ & 2,70 \\
\hline
\end{tabular}

Desgl. mit $10 \%$ speckfett XVI.

\begin{tabular}{|c|c|c|c|c|c|c|}
\hline 3. & $56,2^{0}$ & 52,50 & 3,70 & 4. & 59,30 & $54,7^{\circ}$ \\
\hline 4. & 57,30 & $58,3^{0}$ & 4,00 & 5. & 59,80 & 54,90 \\
\hline 5 & 57,50 & $53,6^{0}$ & 3,90 & 6. & $60,4^{0}$ & $56,0^{0}$ \\
\hline 6. & 58,50 & 54,70 & 3,80 & 7. & 61,00 & 57,20 \\
\hline 7. & 59,50 & 56,20 & 3,30 & 8. & 61,70 & 58,50 \\
\hline 8. & 60,90 & $58,1^{0}$ & 2,80 & 9. & $62,4^{0}$ & 60,00 \\
\hline 9. & 61,30 & $59,3^{0}$ & 2,00 & 10. & $63,1^{1}$ & $60,4^{0}$ \\
\hline 10. & 62,30 & 60,10 & 2,20 & 11. & 63,50 & 60,70 \\
\hline 11. & 62,50 & 60,30 & 2,20 & 12. & 64,10 & 61,20 \\
\hline 12. & 63,80 & $61,6^{\circ}$ & 2,20 & 13. & 64,30 & 61,30 \\
\hline 13. & 64,30 & 62,20 & 2,10 & 14. & 64,70 & 61,60 \\
\hline
\end{tabular}

Nachdem durch die drei ersten Versuchsreihen erwiesen war, daß durch häufiges Umkrystallisieren aus Gemischen von Butterfett mit Schweinefett in den letzten Krystallisationen Glyceride nicht nur mit wesentlich höheren Glycerid-Schmelzpunkten, sondern auch mit größeren Schmelzpunkts-Differenzen erhalten werden als aus reinem Butterfett, erschien die Möglichkeit eines Schweinefettnachweises in Butterfett gegeben.

Bei den drei ersten Versuchsreihen, namentlich bei der dritten, ist jedoch eine so große Zahl von Krystallisationen ausgeführt worden, daf dadurch die praktische Brauchbarkeit des Verfahrens in Frage gestellt werden könnte; wir haben daher in der nachstehenden vierten Versuchsreihe untersucht, wie sich die Verhältnisse gestalten, wenn man die Zahl der Krystallization zur Darstellung der unlöslichsten Glyceride durch Anwendung größerer Mengen der Lösungsmiltel verkleinert.

Vierte Versuchsreihe. Je $50 \mathrm{~g}$ der reinen Butterfette II und IV sowie von Gemisehen dieser mit 10 und $20 \%$ des Schweine-Flomenfettes XII wurden bei Zimmertemperatur zunächst aus $100 \mathrm{ccm}$ Aceton und darauf so oft aus $100 \mathrm{ccm}$ Äther umkrystallisiert, bis die Substanz verbraucht war, d. h. bis nur noch soviel Glyceride ausfielen, wie zur Bestimmung des Schmelzpunktes der Glyceride und zur Darstellnng und Schmelzpunkthestimmung der Fettsäuren erforderlich waren. Dieses war nach 4 bezw. 5 Krystallisationen der Fall. 
Die hierbei erhaltenen Ergebnisse sind in der nachfolgenden Übersicht zusammengestellt und in Fig. 2 graphisch zur Darstellung gebracht.

\begin{tabular}{|c|c|c|c|c|c|c|c|}
\hline $\begin{array}{l}\text { No. der } \\
\text { Krystalli- } \\
\text { sation }\end{array}$ & $\begin{array}{l}\text { Glycerid- } \\
\text { Sclimelzp. } \\
(\mathrm{Sg})\end{array}$ & $\begin{array}{c}\text { Fett- } \\
\text { säuren- } \\
\text { Schmelzp. } \\
\text { (Sf) }\end{array}$ & $\begin{array}{l}\text { Schmelzp." } \\
\text { Differenz } \\
\text { (d) }\end{array}$ & $\begin{array}{l}\text { No. der } \\
\text { Krystalli- } \\
\text { sation }\end{array}$ & $\begin{array}{l}\text { Glycerid- } \\
\text { Schmelzp. } \\
\text { (Sg) }\end{array}$ & $\begin{array}{c}\text { Fett- } \\
\text { säuren- } \\
\text { Schmelzp. } \\
\text { (Sf) }\end{array}$ & $\begin{array}{l}\text { Schmelzp.- } \\
\text { Differenz } \\
\text { (d) }\end{array}$ \\
\hline \multicolumn{4}{|c|}{ Butter II. } & \multicolumn{4}{|c|}{ Butter IV. } \\
\hline 2 & 56,30 & 53,00 & 3,30 & 2 & 56,20 & $52,7^{0}$ & 3,50 \\
\hline 3 & 59,80 & 57,40 & 2,40 & 3 & $59,4^{\circ}$ & 57,20 & 2,20 \\
\hline 4 & 60,60 & 60,10 & 0,50 & 4 & $60,6^{\circ}$ & 60,20 & 0,40 \\
\hline \multicolumn{4}{|c|}{ Desgl. mit $10 \%$ Flomenfett XII. } & \multicolumn{4}{|c|}{ Desgl. mit $10 \%$ Flomenfett XII. } \\
\hline 2 & $58,8^{\circ}$ & 54,10 & 4,70 & 2 & 58,50 & $53,5^{0}$ & 5,00 \\
\hline 3 & 61,30 & 58,30 & 3,00 & 3 & $61,3^{0}$ & 57,50 & 3,80 \\
\hline 4 & $62,3^{\circ}$ & 60,00 & 2,30 & 4 & 62,20 & $60,0^{0}$ & 2,20 \\
\hline \multicolumn{4}{|c|}{ Desgl. mit $20 \%$ Flomenfett XII. } & \multicolumn{4}{|c|}{ Desgl. mit $20 \%$ Flomenfett XII. } \\
\hline 2 & 60,10 & 54,30 & 5,80 & 2 & $60,2^{0}$ & 54,40 & 5,80 \\
\hline 3 & 62,30 & 58,70 & $3,6^{0}$ & 3 & 62,30 & 58,40 & 3,90 \\
\hline 4 & 62,90 & 60,20 & 2,70 & 4 & 63,20 & 60,20 & 3,00 \\
\hline 5 & 63,40 & 60,40 & 3,00 & 5 & 63,40 & $60,4^{0}$ & 3,00 \\
\hline
\end{tabular}

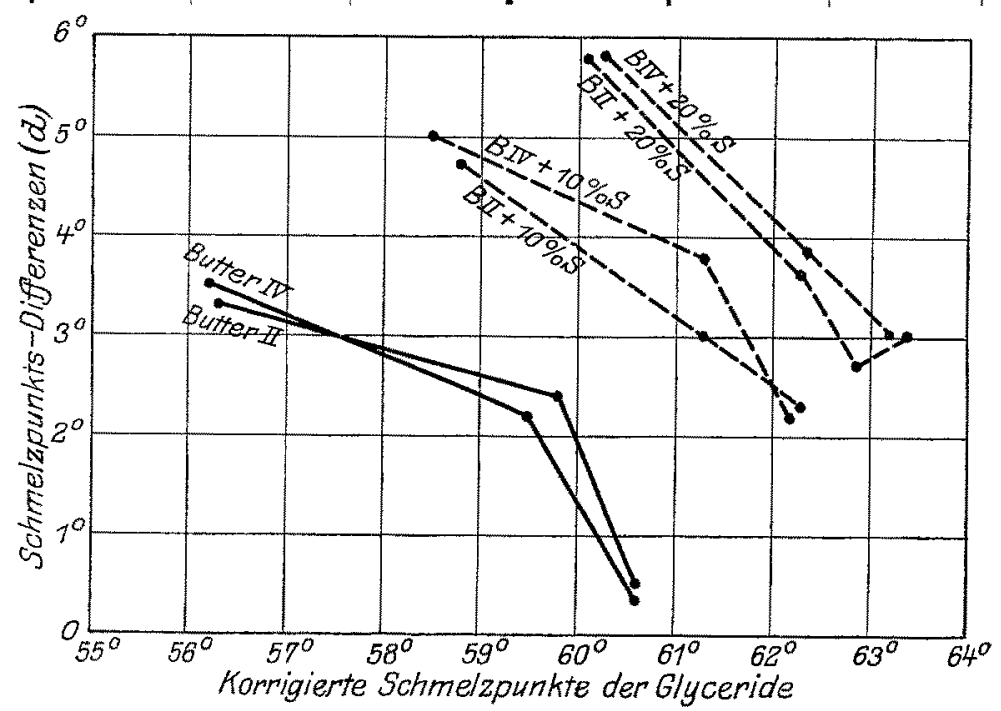

Fig. 2.

Aus den Ergebnissen dieser vierten Versuchsreihe geht zunächst hervor, daß die Schmelzpunkte der schwerlöslichsten Glyceride bei den reinen Butterfetten wesentlich niedriger ausfallen, wenn man durch Anwendung größerer Mengen Lösungsmittel die Zahl der Krystallisationen verringert; denn während wir z. B. bei den reinen Butterfetten II und IV bei der ersten Untersuchung (S. 166) bei 8 Krystallisationen als böehsten Glycerid-Schmelzpunkt $62,4^{\circ}$ fanden, lag der Schmelzpunkt der letzten Krystallisation bei der vierten Versuchsreihe bei $60,6^{\circ}$.

Aus der obigen graphischen Darstellung (Fig. 2) geht hervor, daß aber auch hier bei den Gemischen mit Schweinefett die Schmelzpunkte der unlöslichsten Glyceride 
wesentlich höher liegen und die Schmelzpunkts-Differenzen wesentlich größer sind als bei den zugehörigen reinen Butterfetten.

Die bisher ausgeführten Untersuchungen mit den 4 Butterfetten und ihren Gemischen mit Schweinefett dürften zu der Hoffnung berechtigen, daB es mit Hilfe der Schmelzpunkts-Differenz der unlöslichsten Glyceride gelingt, Zusätze von Schweinefett zu Butter nachzuweisen.

Selbstverständlich bedarf es noch einer Nachprüfung der Befunde an einer größeren Zahl von zuverlässig reinen Butterfetten, auch sog. anormaler Butterfette, ferner einer genauen Festlegung des Untersuchungsverfahrens, ehe die Methode den Fachgenossen zum praktischen Gebrauch empfohlen werden kann. Wir sehen daher, bis dies geschehen ist, auch davon ab, irgendwelche obersten Grenzzahlen für die unlöslichsten Glyceride des reinen Butterfettes anzugeben bezw. vorzuschlagen.

\section{Versuche über den Nachweis von Schweinefett in Gänsefett.}

Vorlänfige Mitteilung über in Gemeinschaft mit H. Merten ausgeführte Versuche.

Das Gänsefett ist in manchen Gegenden Deutschlands ein vielfach begehrter Handelsartikel. Da es meistens sehr weich ist, wird es häufig mit Schweinefett versetzt. Das einzige Verfahren, welches unseres Wissens bisher für den Nachweis dieses Fettes in Gänsefett vorgeschlagen wurde, ist das Polenske'sche Differenzzahl-Verfahren. E. Polenske ${ }^{1}$ ) fand bẹ 18 Proben von reinem Gänsefett die Differenzzahlen 14,7-16,7 und bezeichnet 17 als oberste zulässige Differenzzahl für Gänsefett; er glaubt nach seinem Verfahren 20\% Schweinefett im Gänsefett nachweisen zu können; das Polenske'sche Verfahren scheint bei Gänsefett aber bis jetzt noch nicht nachgeprüft zu sein.

Über die im Gänsefett vorhandenen Glyceride liegt bisher nur eine Untersuchung von J. Klimont und E. Meisels ${ }^{2}$ ) vor, welche durch mehrfaches Umkrystallisieren von Gänsefett aus Aceton mit Zusatz von wenig Chloroform bis zum gleichbleibenden Schmelzpunkt $\left(59^{\circ}\right)$ ein Glycerid mit der Verseifungszahl 203 erhielten, das sie als ein Stearodipalmitin ansehen.

Die günstigen Ergebnisse, welche wir beim Butterfette bezüglich der Nachweisbarkeit von Schweinefett erhalten hatten, veranlaßten uns, auch einige orientierende Versuche darüber anzustellen, ob es durch Darstellung der unlöslichsten Glyceride gelingt, Schweinefett in Gänsefett nachzuweisen.

Es wurden zu diesen Versuchen zwei Proben Gänsefett verwendet, von denen die eine (I) von sehr jungen, wenig fetten Gänsen stammte, während die andere (II) von ausgewachsenen, fetten Gänsen gewonnen war.

Verseifungs - und Jodzahlen der beiden Fette waren folgende:

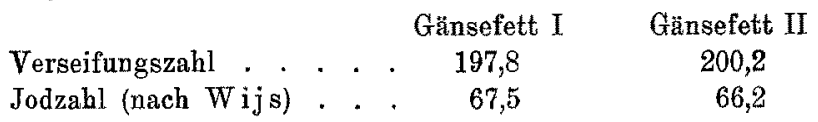

Das Gänsefett I wurde im reinen Zustande und ferner im Gemisch mit $20 \%$ Schweine-Flomenfett dem Krystallisationsverfahren unterworfen. $50 \mathrm{~g}$ beider Fette wurden zunächst 4 -mal aus $50 \mathrm{ccm}$ Aceton und darauf 4-mal aus Äther krystallisiert, worauf bei der letzten Krystallisation nur noch sehr geringe Glyceridmengen sich ausschieden. Die Untersuchungsergebnisse waren folgende:

1) Arb. a. d. Kaiserl. Gesundheitsamte 1907, 26, 444.

2) Monatsh. f. Chemie $1909,30,341-346$. 


\begin{tabular}{|c|c|c|c|c|c|c|c|}
\hline $\begin{array}{l}\text { No. der } \\
\text { Krystalli- } \\
\text { sation }\end{array}$ & $\begin{array}{l}\text { Glycerid- } \\
\text { Schmelzp. } \\
(\mathrm{Sg})\end{array}$ & $\begin{array}{l}\text { Fett- } \\
\text { säuren- } \\
\text { Schmelzp. } \\
\text { (Sf) }\end{array}$ & $\begin{array}{l}\text { Sehmelzp.- } \\
\text { Differenz } \\
\text { (d) }\end{array}$ & $\begin{array}{c}\text { No. der } \\
\text { Krystalli- } \\
\text { sation }\end{array}$ & $\begin{array}{l}\text { Glycerid- } \\
\text { Schmelzp. } \\
\text { (Sg) }\end{array}$ & $\begin{array}{l}\text { Fett- } \\
\text { säuren- } \\
\text { Schmelzp. } \\
\text { (Sf) }\end{array}$ & $\begin{array}{l}\text { Schmelzp.- } \\
\text { Differenz } \\
\text { (d) }\end{array}$ \\
\hline \multicolumn{4}{|c|}{ Reines Gänsefett $I$. } & \multicolumn{4}{|c|}{ Gänsefett I mit $20 \%$ Flomenfett XV. } \\
\hline 3 & 58,00 & 55,20 & 2,80 & 3 & 59,30 & $55,6^{0}$ & 3,70 \\
\hline 4 & 58,90 & 55,80 & 3,10 & 4 & 59,10 & 55,70 & 3,40 \\
\hline 5 & 59,70 & 54,90 & 4,80 & 5 & 60,50 & 56,20 & 4,30 \\
\hline 6 & 59,80 & 5,20 & 4,60 & 6 & 61,10 & $56,5^{0}$ & 4,60 \\
\hline 7 & $60,6^{0}$ & 55,80 & 4,80 & 7 & 62,30 & 56,90 & $5,4^{0}$ \\
\hline 8 & 60,20 & 56,40 & 3,80 & 8 & 68,60 & $59,3^{0}$ & 4,30 \\
\hline
\end{tabular}

Das Gänsefett II wurde sowohl im reinen Zustande als auch in Gemischen mit 10,20 und $30 \%$ Schweine-Flomenfett untersucht.

$50 \mathrm{~g}$ Fett wurden zunächst aus $100 \mathrm{ccm}$ Aceton und darauf so of aus $50 \mathrm{ccm}$ Äther krystallisiert, wie noch Glyceride sich ausschieden. Von der zweiten Krystallisation an wurden die ausgeschiedenen Glyceride in der üblichen Weise untersucht. Die hierbei erhaltenen Ergebnisse sind in der nachfolgenden Übersicht zusammengestellt.

\begin{tabular}{|c|c|c|c|c|c|c|c|}
\hline $\begin{array}{l}\text { No. der } \\
\text { Krystalli- } \\
\text { sation }\end{array}$ & $\begin{array}{l}\text { Glycerid- } \\
\text { Schmelzp. } \\
\text { (Sg) }\end{array}$ & $\begin{array}{c}\text { Fett- } \\
\text { säuren- } \\
\text { Schmolzp. } \\
\text { (Sf) }\end{array}$ & $\begin{array}{l}\text { Schmelzp.* } \\
\text { Differenz } \\
\text { (d) }\end{array}$ & $\begin{array}{l}\text { No. der } \\
\text { Krystalli- } \\
\text { sation }\end{array}$ & $\begin{array}{l}\text { Glycerid- } \\
\text { Schmelzp. } \\
\text { (Sg) }\end{array}$ & $\begin{array}{l}\text { Fett- } \\
\text { säuren- } \\
\text { Sehmelzp. } \\
\text { (Sf) }\end{array}$ & $\begin{array}{l}\text { Schmelzp. } \\
\text { Differenz } \\
\text { (d) }\end{array}$ \\
\hline \multicolumn{4}{|c|}{ Reines Gänsefett II. } & \multicolumn{4}{|c|}{ Desgl. mit $20 \%$ Flomenfett. } \\
\hline 2 & 59,10 & 54,30 & 4.80 & 2 & 59,80 & 55,10 & 3,70 \\
\hline 3 & 60.60 & 5610 & 450 & 3 & $61,2^{0}$ & $57,3^{0}$ & 3,90 \\
\hline 4 & 60,60 & 56.70 & 3,90 & 4 & 61,20 & 57,50 & 3,70 \\
\hline 5 & 61,30 & $57,4^{0}$ & 390 & 5 & 61,80 & 58,20 & 3,60 \\
\hline 6 & 61,30 & 57.60 & 370 & 6 & 63,00 & $58,3^{0}$ & 4,70 \\
\hline & & & & 7 & 63,40 & 58,40 & 5,00 \\
\hline
\end{tabular}

Desgl mit $10 \%$ Flomenfett.

\begin{tabular}{l|l|l|l|l|l|l|l}
2 & 59,30 & 55,10 & 4,20 & 2 & 61,30 & 56,40 & 4,90 \\
3 & 61,10 & 56,30 & 4,80 & 3 & 62,20 & 57,30 & 4,90 \\
4 & 61,10 & 57,10 & 4,00 & 4 & 62,40 & 58,30 & $4,1^{0}$ \\
5 & 61,50 & 58,10 & 3,40 & 5 & 63,40 & 58,30 & 5,10 \\
6 & 61,40 & 58,30 & 3,10 & 6 & 64,30 & 59,50 & 4,80 \\
& & & & & & &
\end{tabular}

Ferner wurde aus einer größeren Probe $(1,5 \mathrm{~kg})$ dieses Gänsefettes II durch 26-maliges Umkrystallisieren aus Äther das unlöslichste Glycerid dargestellt und für dieses der Schmelzpunkt $62,4^{\circ}$ gefunden; die hieraus gewonnen Fettsäuren schmolzen bei $58,2^{0}$, sodaß also die Schmelzpunkts-Differenz $4,2^{0}$ betrug.

Aus diesen vorläufigen Untersuchungen geht hervor, daß die unlöslichsten Glyceride in den Gemischen mit 20 und $30 \%$ Schweinefett einen wesentlich höheren Schmelzpunkt aufweisen als die reinen Gänsefette; dagegen zeigen die SchmelzpunktsDifferenzen zwischen Glyceriden und dazu gehörigen Fettsäuren bei den reinen Gänsefetten und den Gemischen mit Schweinefett keine großen Unterschiede.

Weitere Untersuchungen müssen Aufschluß darüber geben, ob es gelingt, auf Grund des Schmelzpunktes der unlöslichsten Glyceride eine Beimischung von Schweinefett zu Gänsefett zu erkennen. Voraussichtlich wird sich auch hier wie beim Butterfette die Gewinnung der unlöslichsten Glyceride bei Auswahl eines anderen Verhältnisses zwischen Fett und Lösungsmittel noch vereinfachen und abkürzen lassen. 